\title{
Single-footprint retrievals for AIRS using a fast TwoSlab cloud-representation model and the SARTA all-sky infrared radiative transfer algorithm
}

\author{
Sergio DeSouza-Machado ${ }^{1}$, L. Larrabee Strow ${ }^{1,2}$, Andrew Tangborn ${ }^{1}$, Xianglei Huang ${ }^{3}$, Xiuhong Chen ${ }^{3}, \mathrm{Xu} \mathrm{Liu}^{4}$, \\ Wan $\mathrm{Wu}^{5}$, and Qiguang Yang ${ }^{5}$ \\ ${ }^{1}$ JCET, University of Maryland, Baltimore County, Baltimore, Maryland, USA \\ ${ }^{2}$ Department of Physics, University of Maryland, Baltimore County, Baltimore, Maryland, USA \\ ${ }^{3}$ University of Michigan, Ann Arbor, Michigan, USA \\ ${ }^{4}$ NASA Langley Research Center, Langley, Virginia, USA \\ ${ }^{5}$ Science Systems and Applications, Inc, Hampton, Virginia, USA
}

Correspondence: Sergio DeSouza-Machado (sergio@umbc.edu)

Received: 25 July 2017 - Discussion started: 28 July 2017

Revised: 22 November 2017 - Accepted: 27 November 2017 - Published: 25 January 2018

\begin{abstract}
One-dimensional variational retrievals of temperature and moisture fields from hyperspectral infrared (IR) satellite sounders use cloud-cleared radiances (CCRs) as their observation. These derived observations allow the use of clear-sky-only radiative transfer in the inversion for geophysical variables but at reduced spatial resolution compared to the native sounder observations. Cloud clearing can introduce various errors, although scenes with large errors can be identified and ignored. Information content studies show that, when using multilayer cloud liquid and ice profiles in infrared hyperspectral radiative transfer codes, there are typically only 2-4 degrees of freedom (DOFs) of cloud signal. This implies a simplified cloud representation is sufficient for some applications which need accurate radiative transfer. Here we describe a single-footprint retrieval approach for clear and cloudy conditions, which uses the thermodynamic and cloud fields from numerical weather prediction (NWP) models as a first guess, together with a simple cloud-representation model coupled to a fast scattering radiative transfer algorithm (RTA). The NWP model thermodynamic and cloud profiles are first co-located to the observations, after which the $\mathrm{N}$-level cloud profiles are converted to two slab clouds (TwoSlab; typically one for ice and one for water clouds). From these, one run of our fast cloud-representation model allows an improvement of the a priori cloud state by comparing the observed and modelsimulated radiances in the thermal window channels. The re-
\end{abstract}

trieval yield is over $90 \%$, while the degrees of freedom correlate with the observed window channel brightness temperature (BT) which itself depends on the cloud optical depth. The cloud-representation and scattering package is benchmarked against radiances computed using a maximum random overlap (RMO) cloud scheme. All-sky infrared radiances measured by NASA's Atmospheric Infrared Sounder (AIRS) and NWP thermodynamic and cloud profiles from the European Centre for Medium-Range Weather Forecasts (ECMWF) forecast model are used in this paper.

\section{Introduction}

Since the early 2000s, a number of high-spectral-resolution, low-noise, very stable new generation hyperspectral infrared (IR) sounders have been deployed onboard Earth-orbiting satellites, providing daily global top-of-atmosphere (TOA) radiance spectra. In principle these TOA radiances can be inverted to estimate atmospheric temperature and humidity profiles, minor gas concentration, surface temperature and some clouds parameters.

IR sounders have rather large nadir footprints of $\sim 15 \mathrm{~km}$ diameter; consequently, far less than $10 \%$ of scenes are cloud free. Earlier single-footprint retrievals using eigenvalue regression methods have been used with these all-sky (cloud 
and clear) radiances (see for example Weisz et al., 2013) but these methods have no reliable error estimates for individual scenes. Existing NASA and NOAA operational retrieval systems for IR sounders use cloud clearing (Susskind et al., 1998, Susskind et al., 2003, and Gambacorta, 2013). In addition, for any given scene, from a predetermined subset of IR sounder channels, numerical weather prediction (NWP) centers generally only assimilate the radiances that have been deemed unaffected by clouds (Reale et al., 2012; Bauer et al., 2011).

Presently the NASA AIRS operational soundings are performed using cloud-cleared radiances (CCRs) coupled with a clear-sky radiative transfer algorithm (RTA; Susskind et al., 1998, 2003). Cloud-cleared radiances are synthesized using the differences in cloud amounts in a (typically) 3-by-3 set of adjacent fields of view (FOVs) to produce a single effective estimate of the clear-sky radiance. This process increases the retrieval yield (to well above $10 \%$ ) and provides some error estimates but simultaneously reduces the spatial resolution by a factor of 3 . Publicly available products from 1-D variational retrievals include the following:

1. Atmospheric Infrared Sounder (AIRS): NASA, using cloud clearing from a $3 \times 3$ set of footprints (Susskind et al., 1998, 2003).

2. Cross-track Infrared Sounder (CrIS): the NOAA Unique Combined Atmospheric Processing System (NUCAPS), also using cloud clearing from a $3 \times 3$ set of footprints (Gambacorta, 2013).

3. Infrared Atmospheric Sounding Interferometer (IASI): NUCAPS, using cloud clearing from a $2 \times 2$ set of footprints (Gambacorta, 2013).

4. Infrared Atmospheric Sounding Interferometer: EUMETSAT, two-step single-footprint retrievals: piecewise regression for all scenes nominally exploiting IASI in synergy with AMSU+MHS (IASI-only is fallback) followed by a physical retrieval using the optimal estimation method (OEM) (Rodgers, 2000; Steck, 2001) on clear scenes only (IASI-only) (August et al., 2012; EUMETSAT, 2016).

The retrieval approaches mentioned above use various combinations of training to NWP forecasts from the European Centre for Medium-Range Weather Forecasts (ECMWF) either by regression (EUMETSAT IASI) or with neural nets (NASA AIRS) or use climatology (NOAA CrIS). All utilize co-located microwave soundings when possible. The development of a formal error estimate computation in the NUCAPS algorithm is underway (Antonia Gambacorta, personal communication, 2017). The CCR approaches lead to complicated quality control issues, since cloud clearing can fail, and the decisions made in assigning quality flags to the retrievals are not trivial. The cloud-clearing process is especially problematic (Zhou et al., 2005) when the cloud fields are homogeneous and cloud clearing becomes unstable and inaccurate, which introduce errors into retrieved products. This is not necessarily a problem for weather-forecastingoriented applications, since the retrieval quality assurance (QA) can accurately determine when cloud clearing failed. The extensive QA in the AIRS retrieval system deems as many as $\sim 20 \%$ of observations as unsuitable for retrievals. This limits geographic sampling in a complicated way that could make these products problematic for climate statistics. We note here the regression-based single-footprint retrievals provide cloud top information (Weisz et al., 2013); similarly, cloud phase and cirrus effective diameter and optical thickness retrievals are generated at AIRS single-footprint resolution (Kahn et al., 2014) after the L2 thermodynamic retrievals are done - in a separate step that keeps all other retrieval variables constant.

Single-footprint retrievals with hyperspectral sounders provide higher spatial resolution than the $3 \times 3$ cloud-clearing approach, which may be especially significant for water vapor due to its high spatial variability. They also are attractive since you are basing the retrieval on the observed quantity, the level L1b (geolocated and calibrated) radiances, rather than a derived quantity, the cloud-cleared radiances. This requires a fast and reasonably accurate scattering radiative transfer algorithm, where the cloud representation should be simple yet realistic enough to provide useful thermodynamic soundings. Single-footprint retrievals minimize the L1b observed minus computed brightness temperatures (BTs), unlike the AIRS level 2 retrievals (atmospheric products derived from $\mathrm{L} 1 \mathrm{~b}$ radiances) which instead minimize the difference between cloud-cleared radiances and computed brightness temperatures.

Here we examine some viable first steps in performing operational single-footprint retrievals using the OEM for these sensors using a fast scattering RTA that uses a first guess (and a priori estimates) from the ECMWF forecast model. The OEM methodology provides the user with objective diagnostic information, such as error estimates of the retrieved profiles, averaging kernels (AKs) and the information content of the measurements via the degrees of freedom (DOFs). For example we show later in this paper that our single-footprint retrievals have much lower DOFs under thick clouds than in almost clear scenes, which means our retrieval mostly returns the a priori thermodynamic profiles below thick clouds and mainly adjusts the profile above such clouds.

Radiative transfer algorithms for infrared sounders that include scattering by clouds and aerosols are now available (see for example Matricardi, 2005; Liu et al., 2009; De Souza-Machado et al., 2010; Liuzzi et al., 2016). These RTAs use accurate scattering algorithms, but initializing the cloud representation for retrievals is difficult and has not been used operationally.

This paper concentrates on the accuracy of our relatively simple but fast accurate scattering model, especially when coupled with the representation of cloud features in the pro- 
file and initialization of these features in a retrieval. Very few cloud parameters can be retrieved from IR sounder spectra compared to clear-sky geophysical parameters (temperature and humidity), suggesting that simple fast scattering models and cloud representations should be sufficient to radiatively account for cloud and aerosol effects in a retrieval. The paper also demonstrates the utility of using NWP first guess model fields both for thermodynamic and cloud initialization in a high-yield single-footprint physical retrieval, where the computed degrees of freedom are shown to depend on the observed window channel brightness temperature (which itself depends on cloud loading).

In this paper observational data from AIRS are used, while the principal scattering algorithm is the Stand-alone AIRS Radiative Transfer Algorithm (SARTA; Strow et al., 2003) for AIRS. AIRS was designed to provide improved temperature and humidity profiles for NWP and long-term climate studies. The AIRS radiances contain information about the thermodynamic state of the atmosphere (temperature, humidity), trace gases (such as ozone), and surface parameters (Aumann and Pagano, 2002; Strow et al., 2003), as well as ice and water clouds (Kahn et al., 2003, 2005; Wu et al., 2009) and large aerosol particles (mineral dust and volcanic ash) (De Souza-Machado et al., 2010; Clarisse et al., 2010), though we do not consider aerosols in this paper. We introduce our cloud-representation and scattering approach below and test it statistically against an existing RTA, the principalcomponent-based radiative transfer model (PCRTM) (Liu et al., 2006, 2009) that has been supplemented with a full accounting of the cloud subgrid variability (maximum random overlap, or MRO) (Chen et al., 2013).

The PCRTM-MRO implementation (Chen et al., 2013) uses the full vertical cloud profiles in the ECMWF model data. When a 50 sub-column MRO is added to the RTA to represent the cloud subgrid variability, the radiance computation slows by $10 \times$ as compared to a five-sub-column MRO. The appendices show that hyperspectral infrared radiances typically contain $\sim 2-4$ degrees of freedom of cloud information, which could be parametrized by the cloud amount, fraction, and cloud top and bottom pressures. Our approach exploits this to reduce the cloud-representation complexity from $N$-level model cloud fields for cloud ice water content (CIWC) and cloud liquid water content (CLWC), and cloud cover, into two randomly overlapping slabs (hereafter referred to as SARTA-TwoSlab) within the radiative transfer layers, greatly reducing the computational burden. The speed of the scattering calculations are then comparable to those under clear-sky conditions, and we show below that the radiances are as accurate as those from the MRO scheme.

The SARTA-TwoSlab approach is then applied to singlefootprint retrievals for an AIRS granule and compared to the existing NASA AIRS level 2 retrievals. As noted above, a key issue is the proper initialization of the cloud parameters in our RTA. Model fields from ECMWF are used here to initialize the thermodynamic and scattering cloud fields. Although
NWP models do a reasonably good job at estimating cloud parameters, it is very unlikely that the positions of the model clouds are correct on scales near the sounder spatial resolution, especially given the time mismatch between available forecast models and the observations $( \pm 1.5 \mathrm{~h})$. Hence, our cloud parameters are chosen using the closest matches between simulated and observed window region radiances, restricting the choices to model grid points close to the observation. This approach is key to the success of these singlefootprint retrievals.

There are recent papers detailing hyperspectral optimalestimation-based retrievals in the presence of clouds (see for example $\mathrm{Wu}$ et al., 2017; Irion et al., 2017). Our approach is slightly different as it uses easily available NWP fields for initialization and a simple cloud representation which allows for well-defined Jacobians to retrieve thermodynamic profiles and two cloud decks, leading to high yields.

The paper is organized as follows. The AIRS instrument and the use of the ECMWF model are summarized first, followed by a detailed description of the RTA models and the cloud-representation schemes. We then examine the computed radiance differences for both clear-sky and all-sky for these two RTAs and discuss radiance differences arising from perturbations to the TwoSlab cloud-representation schemes. Finally we outline a method to reduce the impact of the spatial-temporal mismatch of observed versus modeled clouds and use this together with the TwoSlab cloud representation to perform single-footprint (cloudy) scene retrievals with a priori thermodynamic profiles and cloud fields from the NWP model fields.

\section{Background}

\subsection{The AIRS instrument and data}

The Atmospheric Infrared Sounder (AIRS) onboard NASA's polar-orbiting Earth Observing System (EOS) Aqua platform has 2378 channels, covering the thermal infrared (TIR; roughly $649-1613 \mathrm{~cm}^{-1}$ ) and shortwave infrared (2181$2665 \mathrm{~cm}^{-1}$ ) spectral range. The full widths at half maximum satisfy $v / \delta v \sim 1200$. The (spectral dependent) noise is typically $\leq 0.2 \mathrm{~K}$ at $250 \mathrm{~K}$ scene temperatures. The instrument, operational since September 2002, is expected to continue operating until the early 2020s. AIRS has a $13.5 \mathrm{~km}$ nadir footprint from a $\sim 705 \mathrm{~km}$ orbit and scans about $\pm 49.5 \mathrm{de}-$ grees from nadir. Radiances from AIRS have been shown to be very stable and accurate (Aumann et al., 2006).

About 1500 AIRS channels that have remained stable over the life of the AIRS mission were selected for this paper. This was done by examining the statistics of the 14+ time series of AIRS radiances (of all channels) in the AIRXBCAL clear-sky data set (ocean scenes only), which contains scenes deemed to be clear for each day. More details about this channel list can be obtained from 
the authors. NEDT (noise equivalent delta temperature) values used in this paper come from the v9.5.0 (1 July 2011) file available at https://disc.gsfc.nasa.gov/information/ documents?title=AIRS\%20Documentation.

\subsection{The ECMWF model fields}

The core ECMWF 0-10 day forecasts are produced using the Integrated Forecasting System (IFS) (Uppala et al., 2005; Dee et al., 2011). The topmost ECMWF level is $0.01 \mathrm{mb}$, with terrain following $\sigma$ levels from the surface to $0.01 \mathrm{mb}$. The level spacing is finest in the boundary layer and coarsest at the top. At each model grid point, the cloud fields include the cloud ice water and cloud liquid water content profiles $\left(\operatorname{CIWC}(z), \operatorname{CLWC}(z)\right.$ in $\left.\mathrm{g} \mathrm{g}^{-1}\right)$, cloud cover profile $(\mathrm{CC}(z))$ and total cloud cover (TCC).

Here we use 91 level ECMWF model fields, at a horizontal resolution of $0.25^{\circ}$ (about $14 \mathrm{~km}$ at the equator, approximately the same size as the $13.5 \mathrm{~km}$ AIRS nadir footprint). AIRS is on a 13:30 equator ascending overpass orbit while ECMWF analysis and forecast are output at $3 \mathrm{~h}$ intervals (eight model outputs per day) starting at 00:00 GMT. The forecast and analysis output that is closest in time is used to provide gridded fields, which are matched (using nearest grid point) to the AIRS L1B observations. This means the profile versus observed matchups are within $0.25^{\circ} \pm 0.05^{\circ}$ of each other, while the time differences are uniformly distributed within $\pm 1.5 \mathrm{~h}$.

The topmost AIRS RTA pressure layer boundary is $0.005 \mathrm{mb}$, so US standard temperature, water vapor and ozone fields (McClatchey et al., 1972) are appended above the $0.01 \mathrm{mb}$ boundary. Standard profiles are also used for the remaining atmospheric gases, with carbon dioxide $\left(\mathrm{CO}_{2}\right)$ and methane $\left(\mathrm{CH}_{4}\right)$ concentrations set to 385 and $1.8 \mathrm{ppmv}$ at the surface. Masuda ocean model emissivities (Masuda et al., 1988) are used, while land emissivities come from (Zhou et al., 2011).

\subsection{Radiative transfer models}

The description of existing cloud-representation and scattering codes for nadir infrared sounders include those found in (Zhou et al., 2005), (Liu et al., 2009), (Chen et al., 2013), (Ou et al., 2013), (Vidot et al., 2015), (Liuzzi et al., 2016), and (Griessbach et al., 2013) for infrared limb sounders; separate examples can also be found for dust and volcanic ash aerosols.

We use two different RTAs, described below, to simulate AIRS infrared radiances that differ primarily in the scattering radiative transfer. Both RTAs use the same AIRS 100 pressure layer scheme (Strow et al., 2003); layer thicknesses range from $0.25 \mathrm{~km}$ at the surface, $0.75 \mathrm{~km}$ at the upper tropopause and about $4 \mathrm{~km}$ at $0.005 \mathrm{mb}$ (about $80 \mathrm{~km}$ ), which is the TOA radiance for the model.

\subsubsection{SARTA}

The clear-sky version (with gray cloud capability) of SARTA is used for the NASA AIRS level 2 retrievals. Layer optical depths are generated using precomputed predictor coefficients (Aumann and Pagano, 2002; Strow et al., 2003, 2006). SARTA is trained using optical depths from the pseudoline-by-line kCompressed Atmospheric Radiative Transfer Algorithm (kCARTA) package (De Souza-Machado et al., 2002). SARTA has been validated during dedicated AIRS campaigns (Strow et al., 2006).

We extended SARTA to handle clouds and aerosols, based on the parametrization for cloud longwave scattering for use in atmospheric models (PCLSAM; Chou et al., 1999) algorithm. The PCLSAM algorithm recasts the extinction, single scattering albedo and asymmetry factor due to clouds and aerosols into an effective absorption optical depth and is used in other infrared fast models (see for example Matricardi, 2005; Vidot et al., 2015; Liuzzi et al., 2016). For each SARTA AIRS layer that contains a cloud/aerosol, the total optical depth is then the sum of the atmospheric gas optical depth plus the cloud/aerosol effective optical depth. Fast, efficient clear-sky radiative transfer can then be used to compute the TOA radiance and to compute finite-difference Jacobians. Cirrus cloud scattering parameters come from (Baum et al., 2007, 2011), while water cloud scattering parameters are computed using Mie scattering coefficients and using water refractive indices from the Optical Properties of Aerosols and Clouds (OPAC) database (Hess et al., 1998). The parameters are integrated over a modified gamma droplet size distribution of effective variance 0.1 (dimensionless) and effective radius (typically) of $20 \mu \mathrm{m}$.

\subsubsection{PCRTM}

We benchmarked the SARTA-TwoSlab model versus the radiance simulator based on the principal-component-based radiative transfer model (PCRTM version 2.1) (Liu et al., 2006, 2009) with full accounting of the cloud subgrid variability (Chen et al., 2013). PCRTM is a fast model that computes atmospheric optical depths based on the line-by-line radiative transfer model (LBLRTM; Liu et al., 2006). PCRTM calculates reflectance and transmittance of water and ice clouds using a parametrization scheme (Liu et al., 2009) based on a look-up-table trained using the 32-stream Discrete Ordinates Radiative Transfer Program for a Multi-Layered PlaneParallel Medium (DISORT; Stamnes et al., 1988) and using single scattering properties calculated by Yang et al. (2002), Wei et al. (2004), Huang et al. (2004), and Niu et al. (2007). Again, ice scattering coefficients come from (Baum et al., 2011), while the refractive indices for water come from (Segelstein, 1981). Unlike conventional channel-based radiative transfer models which compute the radiance of each channel separately, the PCRTM calculates the scores (i.e., the coefficients) of precomputed principal components (PCs) in 
the spectral domain, with the instrument spectral response function taken into account. The PC scores contain essential information about the radiances and can be calculated by performing monochromatic radiative transfer calculations at a small number of frequencies. The spectral radiances are then computed by multiplying the PC scores with precomputed PCs. With this approach, the PCRTM achieves both high accuracy and extremely fast computational and high storage efficiency (Liu et al., 2006; Chen et al., 2013). (Chen et al., 2013) showed that the root-mean-square differences between the PCRTM and LBLRTM (a widely used line-by-line radiative transfer benchmark model, Clough et al., 2005) are $0.67 \mathrm{~K}$ for the clear-sky case and $0.78 \mathrm{~K}$ for the overcast case, for a wavenumber range spanning $0-2000 \mathrm{~cm}^{-1}$. (Chen et al., 2013) implemented a radiance simulator using the PCRTM and taking cloud variability into account in the same way the ISSCP simulator does (Klein and Jakob, 1999).

\section{Cloud model field conversion}

Here we describe the TwoSlab cloud-representation and the MRO cloud models. The latter is used exclusively with PCRTM and the former with SARTA except in Sect. 4.2 when both PCRTM and SARTA use the TwoSlab model for inter-RTA comparison purposes. The MRO model has been previously documented and is briefly summarized at the end of this section.

\subsection{TwoSlab conversion}

Our cloud-representation scheme replaces the $N$-level NWP cloud vertical profiles by one or two randomly overlapping finite width slabs clouds. The NWP cloud liquid water and cloud ice water content (CLWC, CIWC) profiles (in $\mathrm{g} \mathrm{g}^{-1}$ ) are integrated to obtain the column loading of the clouds (in $\mathrm{g} \mathrm{m}^{-2}$ ) and also to determine the slab cloud top and bottom pressures. The CIWC and CLWC profiles, cloud cover profiles and total cloud cover are used to determine the slab cloud fractions. Effective particle sizes then need to be assigned to the clouds.

Infrared sensors cannot see through optically thick clouds and are mostly sensitive to the emission from the cloud upper boundary while emission throughout the cloud can contribute to the outgoing radiance for less optically thick clouds. The TwoSlab model is very flexible when placing the slabs, for example (a) at the weighted mean or centroid (C) of the cloud ice or cloud liquid profile or (b) near the most prominent cloud profile peak $(\mathrm{P})$, which is best for optically thin clouds.

In practice, the cloud content profile $\mathrm{CXWC}(z)$ (where $X=I, W$ for ice or water cloud) is first smoothed in order to make it easier to localize the positioning of the (ice or water) cloud slabs. The NWP cloud profiles usually result in the code identifying one water and one ice cloud, though the
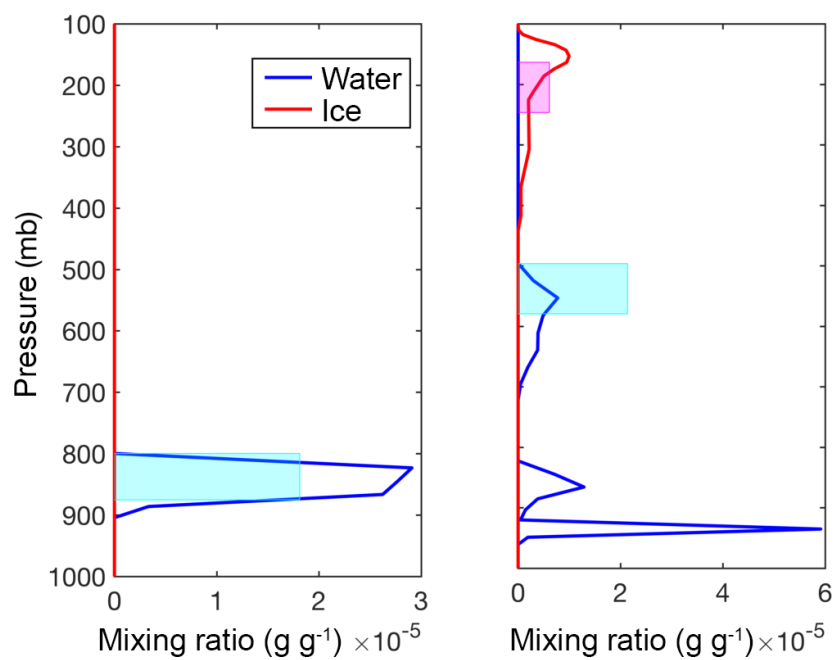

Figure 1. Example of cloud vertical profiles, reduced to one or two slabs. The red and blue curves come from the NWP model, while the cyan and magenta bars are the resulting locations (and loadings) for the slabs.

CLWC-CIWC pairs could produce two liquid or two ice slab clouds, or just a single slab cloud.

Figure 1 shows two examples of slab cloud outputs. The left panel is the simpler case where the NWP cloud profile (in blue) is singly peaked. The right panel shows a case where the profiles are much more complex, and the cyan (water cloud) slab is placed higher in the atmosphere closer to the peak of the weighting function. The integrated cloud amount $\mathrm{g} \mathrm{m}^{-2}$ is proportional to the width of the cyan and magenta bars.

Assuming one ice and one water cloud slab are produced, the cloud fractions are constrained as follows:

$\mathrm{TCC}=c_{\text {water }}+c_{\text {ice }}-c_{\text {overlap }}$,

where TCC is the NWP total cloud cover. From this we compute the individual clouds fractions and their overlap using the following criteria:

1. If there is only one cloud present, its cloud fraction is set to TCC.

2. For two ice or two water clouds, the fraction for the first cloud $c_{1}$ is set as TCC $\times R$, where $R$ is a random number between 0 and 1 . Then a check is performed to see if TCC is less than a random number; if so, $c_{\text {overlap }}$ is set to be $c_{1} \times R R$, where $R R$ is also random; otherwise, the overlap fraction is set to $0 . c_{2}$ then follows from Eq. (1).

3. If there is one ice and one water cloud, the cloud fractions are set according to $c_{\text {water }}, c_{\text {ice }}$ (described below); after that $c_{\text {overlap }}$ is set using Eq. (1).

For the third case, the water cloud slab fraction comes from weighting the NWP cloud cover (CC) profile using 
the cloud liquid water content profile $c_{\text {water }}=\sum \operatorname{CLWC}(z) \times$ $\mathrm{CC}(z) / \sum \operatorname{CLWC}(z)$. The ice cloud fraction $c_{\text {ice }}$ is similarly determined.

Water cloud droplet effective diameters vary with season and geographic location (King et al., 2013); to model this we use an effective diameter of $20 \mu \mathrm{m}$ plus a uniformly distributed random offset. The ice effective particle size is estimated from a temperature-based parametrization by $\mathrm{Ou}$ and Liou (1995), where the NWP temperature profile is used to associate the ice cloud slab top pressure with a cloud top temperature.

\subsection{Radiance computation}

The $i$ th channel all-sky radiance $r_{i}(v)$ is computed using four weighted radiance streams:

$$
\begin{aligned}
r_{i}(v)= & f_{\mathrm{clr}} r_{i}^{\mathrm{clr}}(v)+c_{\text {overlap }} r_{i}^{(12)}(v) \\
& +c x_{1} r_{i}^{(1)}(v)+c x_{2} r_{i}^{(2)}(v),
\end{aligned}
$$

where $f_{\text {clr }}$ is the clear fraction; $c x_{j}, j=1,2$ is the exclusive cloud slab $j$ fraction; and $c_{\text {overlap }}$ is the cloud overlap between the two cloud slabs - the exclusive cloud fraction being related to the cloud fraction via the relationship $c x_{j}=$ $c_{j}-c_{\text {overlap }}$. The model currently exclusively uses ice or water clouds when computing the radiances $r_{i}^{(1)}(v), r_{i}^{(2)}(v)$ associated with the cloud slabs; $r_{i}^{\text {clr }}(\nu)$ is the clear-sky contribution while $r_{i}^{(12)}(v)$ is the radiance contribution from the cloud overlap. Since the atmospheric gas optical depth computation dominates the runtime, computing four radiance streams is not a speed penalty, and the overall average runtime per profile is about double that for a single clear-sky radiance computation.

\subsection{Maximum random overlap conversion}

The MRO cloud processing for the PCRTM model is described in (Chen et al., 2013) and will only be briefly summarized here. MRO converts the NWP water and ozone level profiles to 100-layer profiles. For each layer, the cloud ice water content and cloud liquid water content mixing ratios are converted to a cloud optical depth. The optical depths at each layer are summed. Layers above $440 \mathrm{mb}$ are considered ice clouds, and layers in the lower atmosphere are assigned to water clouds (Rossow and Schiffer, 1983, 1991). The effective water diameter is set at $20 \mu \mathrm{m}$ while the effective ice diameter is again temperature dependent, based on the parametrization in (Ou and Liou, 1995). The cloud cover profile $\operatorname{cc}(z)$ is used to generate 50 sub-columns using MRO (Chen et al., 2013) for which one radiance is computed per sub-column; the final radiance is an average over these subcolumns.

\section{Inter-comparisons of SARTA and PCRTM}

\subsection{Clear-sky comparisons}

An earlier inter-comparison of the SARTA and PCRTM clear-sky models is presented in (Saunders et al., 2007). In this subsection we assess the more recent spectroscopy embedded in the SARTA and PCRTM codes, using ECMWF thermodynamic profiles and surface parameters to compare clear-sky radiances computed from the models.

We use 1600 randomly chosen nighttime scenes observed by AIRS on 1 March 2009 for an inter-RTA clear-sky simulation comparison. The locations span all climate zones over ocean and land, as well as all AIRS scan angles. Nighttime scenes are used to avoid non-local thermodynamic equilibrium (De Souza-Machado et al., 2007) and solar surface reflectivity during the daytime in the $4 \mu \mathrm{m}$ shortwave region. Both of these effects are handled differently by SARTA and PCRTM and are not relevant to this paper.

Figure 2 shows the calculated BT biases between SARTA and PCRTM clear-sky models along with AIRS noise levels. The top panel shows the mean differences, while the bottom shows the standard deviations. The mean bias between SARTA and PCRTM clear calculations is within AIRS noise levels at all channels, except in the methane region $\left(1300 \mathrm{~cm}^{-1}\right)$ and some channels in the water vapor $6.7 \mu \mathrm{m}$ region. This is due to differing methane and water vapor spectroscopy and continuum models in these two RTAs. In addition, PCRTM uses a density-weighted layer temperature that may introduce differences. Overall, differences between SARTA and PCRTM effective BTs are typically within AIRS noise levels.

\subsection{All-sky comparisons for TwoSlab clouds}

Here we compare all-sky radiances computed using SARTA and PCRTM but use the same TwoSlab cloud representation in both RTAs. This tests the differences in each RTA's underlying scattering algorithm by keeping the cloud representation the same in both. Thus this directly compares the relative accuracy of the PCLSAM scattering algorithm used in SARTA against the DISORT-based scattering used in PCRTM.

The PCLSAM algorithm approximations in SARTA are more accurate for absorptive clouds that are more likely in the mid-IR. However, the DISORT-based scattering in PCRTM is more accurate if the cloud representation is correct. In general it would be reasonable to expect the differences to increase with optical depth and/or cloud fraction. In addition, in the TIR the single scattering albedo of water clouds is generally larger than that of ice, so we would also expect larger differences for water clouds.

To evaluate the SARTA (PCLSAM) versus PCRTM (DISORT) radiance differences, we used 1000 scenes maximizing cloud variability and spanning all climate types from 

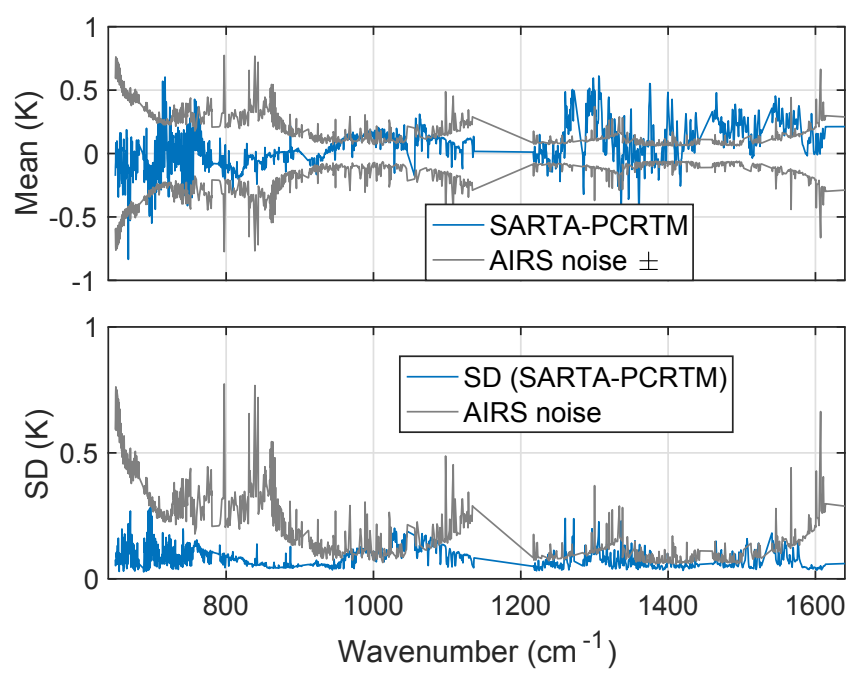

Figure 2. Spectral differences for clear-sky calculations between SARTA and PCRTM for ocean night scenes. Also shown are the AIRS noise levels. Typical difference between PCRTM and SARTA are less than $0.25 \mathrm{~K}$, except in the methane region $\left(1300 \mathrm{~cm}^{-1}\right)$ where SARTA does not use line mixing and in some of the strong water vapor lines $\left(1400+\mathrm{cm}^{-1}\right)$.

AIRS on 1 March 2009 (see Appendix C). After matching the thermodynamic and cloud NWP fields to the observations, and subsequent conversion of the input cloud profiles to slab clouds, SARTA and PCRTM were run twice: (a) a clear-sky run where no cloud effects are included and (b) an all-sky run using the TwoSlab cloud representation derived from ECMWF.

In the TIR window region, cloud forcing (difference between observed BT and surface temperature) can be as large as $100 \mathrm{~K}$ (for the deep convective cloud, DCC, cases). For our sample set the mean (AIRS observation-SARTA-TwoSlab RTA simulation) difference at $820 \mathrm{~cm}^{-1}$ is $-1.8 \pm 21.8 \mathrm{~K}$ compared to a mean cloud effect of $27.9 \mathrm{~K}$. Similarly, at $1231 \mathrm{~cm}^{-1}$ the mean difference is $-2.4 \pm 20.1 \mathrm{~K}$ compared to a mean cloud effect of $28.7 \mathrm{~K}$. The corresponding biases for the PCRTM TwoSlab simulations are $-2.4 \pm 21.9 \mathrm{~K}$ at $820 \mathrm{~cm}^{-1}$ and $-2.6 \pm 19.5 \mathrm{~K}$ at $1231 \mathrm{~cm}^{-1}$. Later in this section we further utilize the $1231 \mathrm{~cm}^{-1}(8.12 \mu \mathrm{m})$ channel to compare the observed versus computed radiances since it is largely free of atmospheric absorption except for several degrees of water vapor forcing in the tropics near the surface, but at the same time it is strongly impacted by clouds.

The effects of the clouds become less noticeable for channels sensing high in the atmosphere, such as the 650$700 \mathrm{~cm}^{-1}$ and $1400-1600 \mathrm{~cm}^{-1}$ regions. These comparisons show the differences between the scattering RTAs are much smaller than the mean cloud effect.

Figure 3 shows the mean and standard deviations between SARTA-TwoSlab and PCRTM-TwoSlab using double differences, where the mean of the clear-sky differences is re-

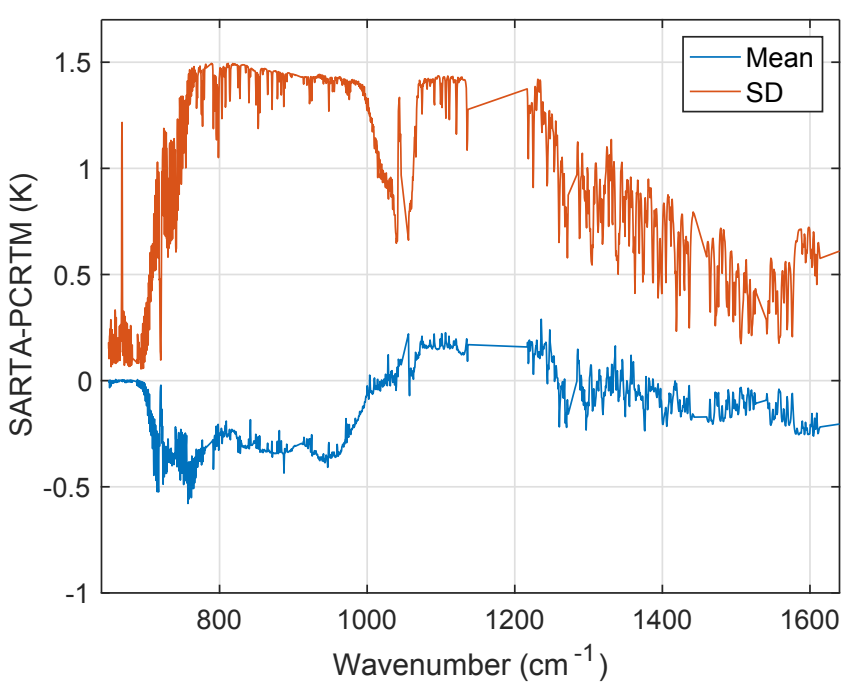

Figure 3. Biases and standard deviations between 1000 TwoSlab computations using SARTA and PCRTM. See the text for details on these double-difference signals.

moved from the mean of the all-sky differences. The double difference removes residual spectroscopic differences, allowing one to attribute the remaining differences to the RTA scattering algorithms. Differences are seen in the window where cloud scattering is most significant, but overall they are less than $0.5 \mathrm{~K}$. A detailed examination showed that the differences for ice clouds were proportional to the ice cloud fraction, while there was comparatively more scatter in the differences between PCLSAM and DISORT for water clouds at any cloud fraction.

These comparisons indicate that our implementation of the PCLSAM model is a fast yet simple and effective method to include scattering effects in the TIR, as has also been shown by Matricardi (2005), Vidot et al. (2015), and Liuzzi et al. (2016).

\section{All-sky comparisons: TwoSlab versus MRO}

We now compare radiances produced using the TwoSlab cloud-representation model using SARTA and those produced using the MRO cloud representation using PCRTM, comparing both to AIRS all-sky observations. The AIRS data obtained on 11 March 2011 are used in this section, with colocated thermodynamic and cloud fields from the ECMWF model. The SARTA-TwoSlab calculations used slab clouds at the weighted mean (centroid) of the cloud profiles. An important factor in the comparisons to actual AIRS observations is the $\pm 1.5 \mathrm{~h}$ mismatch between NWP forecast output and AIRS observations at 01:30 and 13:30 equator crossing time, which implies the cloud locations in the model are likely to be in the wrong position. 

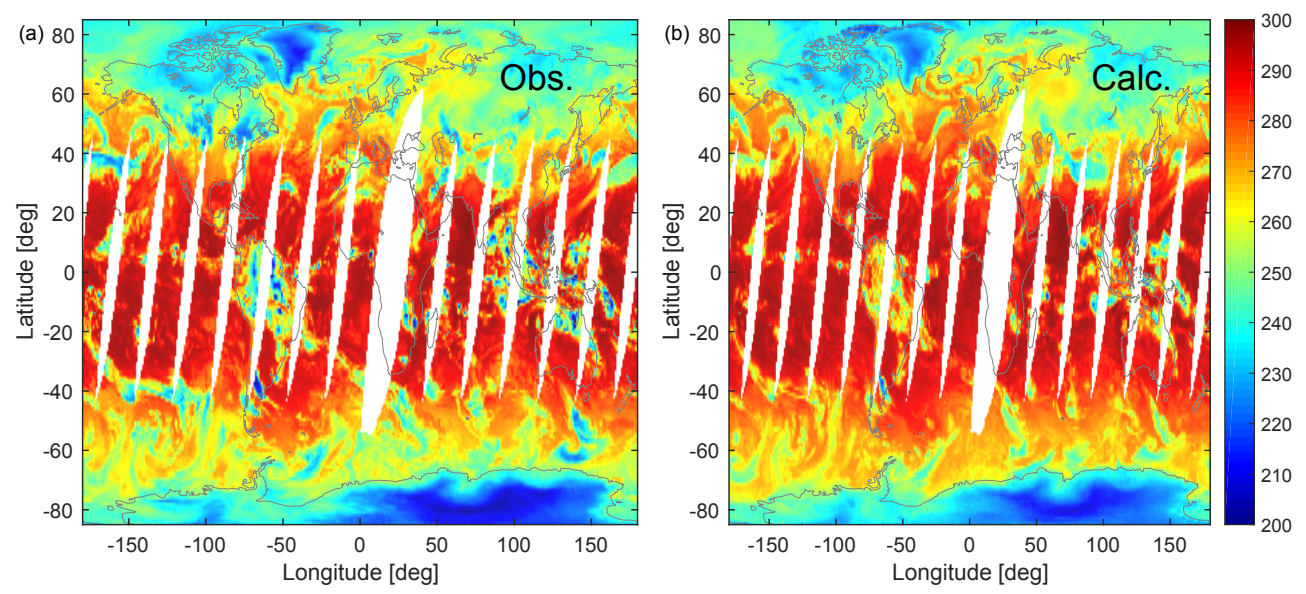

Figure 4. (a) AIRS $1231 \mathrm{~cm}^{-1}$ channel brightness temperatures (in K) for the descending node (night) on 11 March 2011 , using a 1 degree grid. (b) Computed BT 1231 using SARTA-TwoSlab. Note the excellent agreement between the observations and calculations - there are large areas in the tropics with $\sim 0 \mathrm{~K}$ differences. Careful examination (of obs.-calc.) does reveal large negative and positive biases scattered throughout due to spatial mismatches in the model versus observed clouds, for example the tropical west Pacific (see Fig. 5) and the Amazon.

The left panel of Fig. 4 shows the $1231 \mathrm{~cm}^{-1}$ BTs for the nighttime overpasses on 11 March 2011. The data are averaged over a 1 degree grid for plotting purposes. The white areas are gaps between the $\sim 2000 \mathrm{~km}$ wide AIRS swaths. The right panel shows the BT $1231 \mathrm{~cm}^{-1}$ calculated with SARTA-TwoSlab. The observations in the left panel show that the BTs vary from $330 \mathrm{~K}$ (hot surface, no clouds) to as low as $210 \mathrm{~K}$ for deep convective clouds in the tropics. Over tropical oceans, most of the pixels in the right-hand panel agree very well with the observations, indicating almost zero difference between observations and calculations generally in regions with few clouds. Conversely, the Tropical Warm Pool (TWP) and the Amazon show spatial differences due to small temporal and spatial errors in the model, especially for deep convective clouds which have extremely cold cloud tops; in particular note the almost total lack of DCC over the Amazon compared to the observations. However, these results show that overall the dynamical distribution of moisture and clouds is well represented by ECMWF as noted by Allan et al. (2005) and Shahabadi et al. (2016).

Figure 5 is a zoom of the Tropical Warm Pool region. Extremely cold cloud tops are clearly seen by AIRS (left panel). The right panel plots the differences between observed and computed all-sky radiances, clearly showing unsurprising offsets between observed and computed convective structures, which could be due to both model errors and to the $\pm 1.5 \mathrm{~h}$ time offset between observations and model fields. The rapid varying spatial differences in these biases of opposite sign suggests that the model clouds are relatively accurate, they just have slightly different spatial patterns.

Plots of the $1231 \mathrm{~cm}^{-1}$ all-sky calculations using PCRTM-MRO are very similar to Figs. 4 and 5, namely large areas of the tropical oceans having almost zero bias and with noticeable mismatches of cold cloud tops.

\subsection{Window channel PDFs}

Here we explore the similarities and differences between the observations, SARTA-TwoSlab and PCRTM-MRO by examining the radiance probability distribution functions (PDFs) and the scene dependence of the mean BT differences, again for the $1231 \mathrm{~cm}^{-1}$ AIRS channel.

Cloud mismatch errors will contribute to a significant portion of the standard deviation between observations and calculations, as is evident from Figs. 4 and 5. Conversely, as mentioned earlier, the dynamic range spanning 200 to $330 \mathrm{~K}$ is seen both in observations and calculations from both cloud-representation models. The left panel of Fig. 6 is a plot of the corresponding histograms or un-normalized probability distribution functions; the bins are $1 \mathrm{~K}$ wide. The curves are the nighttime observations (black), SARTA-TwoSlab calculations (blue and cyan) and PCRTM-MRO calculations (red). The blue and cyan SARTA-TwoSlab calculations differ in the positioning of the slabs: the peak of the cloud weighting function or the centroid of the cloud profile respectively, as described in Sect. 3.1. The calculated radiance histograms are very similar compared to their differences with the observations. If the $1231 \mathrm{~cm}^{-1}$ PDFs are subset for different geographical regions, discrepancies between the computed and observed PDFs are easier to evaluate compared to the global PDFs. For example we discern the following four points (marked in the left-hand panel):

1. In the tropics the observations have more cold (DCC) scenes, also seen in (Shahabadi et al., 2016).

2. The frozen oceans in the northern polar regions and off the Antarctic coast have BT1231 calculations between 240 and $260 \mathrm{~K}$, which are quite different than what is observed. 

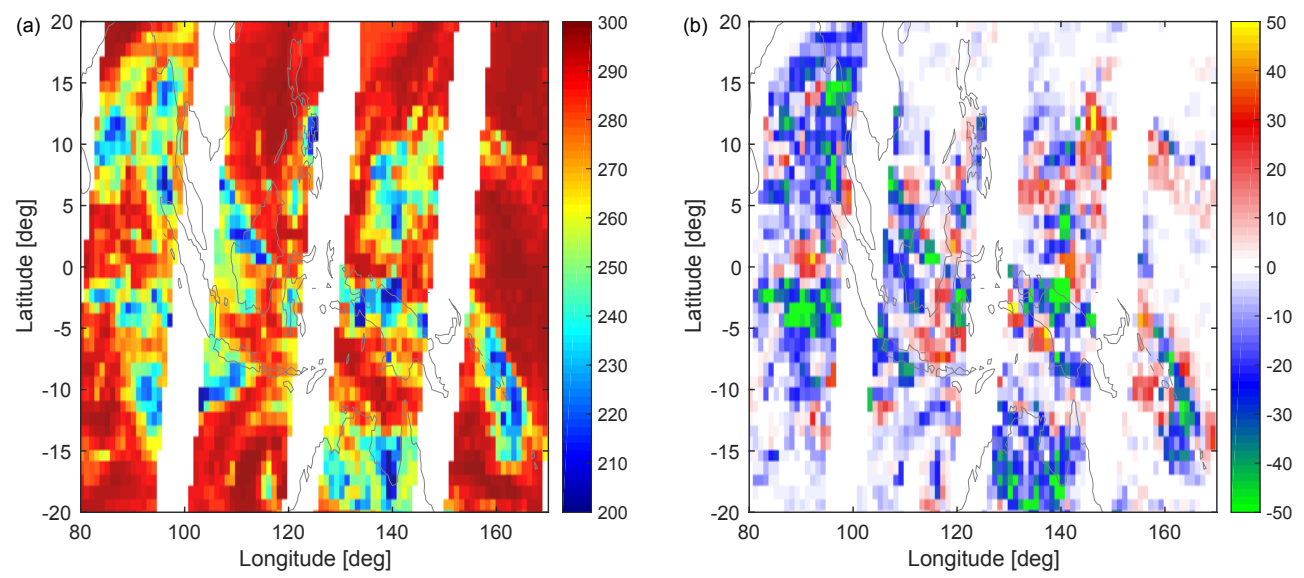

Figure 5. (a) Zoom of left panel in Fig. 4 in the tropical western Pacific region. (b) Zoom of the model biases highlighting that most large biases are due to small spatial offsets of the model clouds. Note that red and blue pixel are often adjacent to each other. For the region shown, the mean bias and standard deviation of (observations - SARTA-TwoSlab) is $-6.1 \pm 20.1 \mathrm{~K}$.

3. A significant portion of the BT1231 calculations between 260 and $280 \mathrm{~K}$ come from the extra-tropical oceans $\left(-40\right.$ to $-70^{\circ} \mathrm{S}$ and +40 to $\left.+60^{\circ} \mathrm{N}\right)$.

4. The whisker plots (circles are means while the bars are the standard deviation) show the calculations are generally slightly warmer than the observations, while the spreads are all very similar.

The right panel of Fig. 6 shows the mean difference between various pairs of observations and calculations as a function of the scene radiative temperature, BT1231. The abscissa is constrained to be 205 to $300 \mathrm{~K}$, as the low number of observations outside these limits yields large average differences. Typical standard deviations are $\pm 10 \mathrm{~K}$ for the blue, cyan and red (obs.-calc.) curves and $\pm 5 \mathrm{~K}$ for the green and black (inter-model) curves. The calculations agree well with observations for high BT1231 (280-300 K) values where clouds are less important. As the scene temperature decreases, the observations indicate a lack of high clouds in the model, which has been noted previously (see for example Shahabadi et al., 2016). This panel magnifies the differences shown in the left-hand panel. For example when the observed clouds are cold (high clouds), one would expect placing the (ice) slab cloud as high as possible (P) would produce a smaller bias than if you placed the slab cloud lower down in the atmosphere at the centroid (C). Indeed this is clearly seen in the right-hand panel - the $(\mathrm{P})$ bias in blue for the cold clouds (BT $1231<250 \mathrm{~K}$ ) is noticeably less than the (C) bias shown in cyan. The differences between SARTA (P and C) and PCRTM-MRO are generally small compared to the observation versus model differences.

Note that for the highest clouds SARTA (C) is hotter than SARTA (P), which makes sense since these are very optically thick clouds and the TIR weighting function peaks at the cloud top. For warm scenes, the MRO simulations are again closer to SARTA (P) than SARTA (C), indicating that, as expected, placing clouds near the weighting function peak in the TwoSlab algorithm is preferable to the centroid.

Table 1 summarizes the $1231 \mathrm{~cm}^{-1}$ window channel global statistical comparisons including differences with observations and differences among the various RTAs. First note that the high standard deviations for observations minus computed radiances are dominated by spatial-temporal mismatches and are not necessarily indicative of model limitations. For the purposes of this paper, the most interesting result is that the SARTA (P) agrees better with observations in the mean, while SARTA (C) generally agrees better with MRO. The uncertainties in the model cloud fields are sufficiently large (especially given spatial-temporal mismatches) that these comparisons are not sufficient to indicate which scattering model is more accurate.

We note the PDF correlations between observations and all the calculations and also among the calculations themselves are typically 0.9 and above, which reinforces the point that the NWP fields from ECMWF capture much of the atmospheric variability that is observed; however, the mismatch between observed and model cloud tops led to biases on the order of $2-4 \mathrm{~K}$ with standard deviations on the order of $10 \mathrm{~K}$.

The implications of this are that, by shifting the position of the model clouds, one could significantly mitigate the differences, and hence have a priori micro-physical properties of the TwoSlab clouds, for a physical single-footprint all-sky retrieval. This idea is exploited later in the section on applying the TwoSlab code for use in single pixel all-sky retrievals.

We close this subsection with Fig. 7, where the BT1231 observations and calculations are plotted as normalized PDFs for the tropics, midlatitudes and polar regions. Note that we have limited the PDFs to contain data only over nonfrozen oceans, using the ECMWF sea ice fraction model field. As in Fig. 6 the black curves are observations, while SARTA-TwoSlab peak and centroid calculations are in (blue 

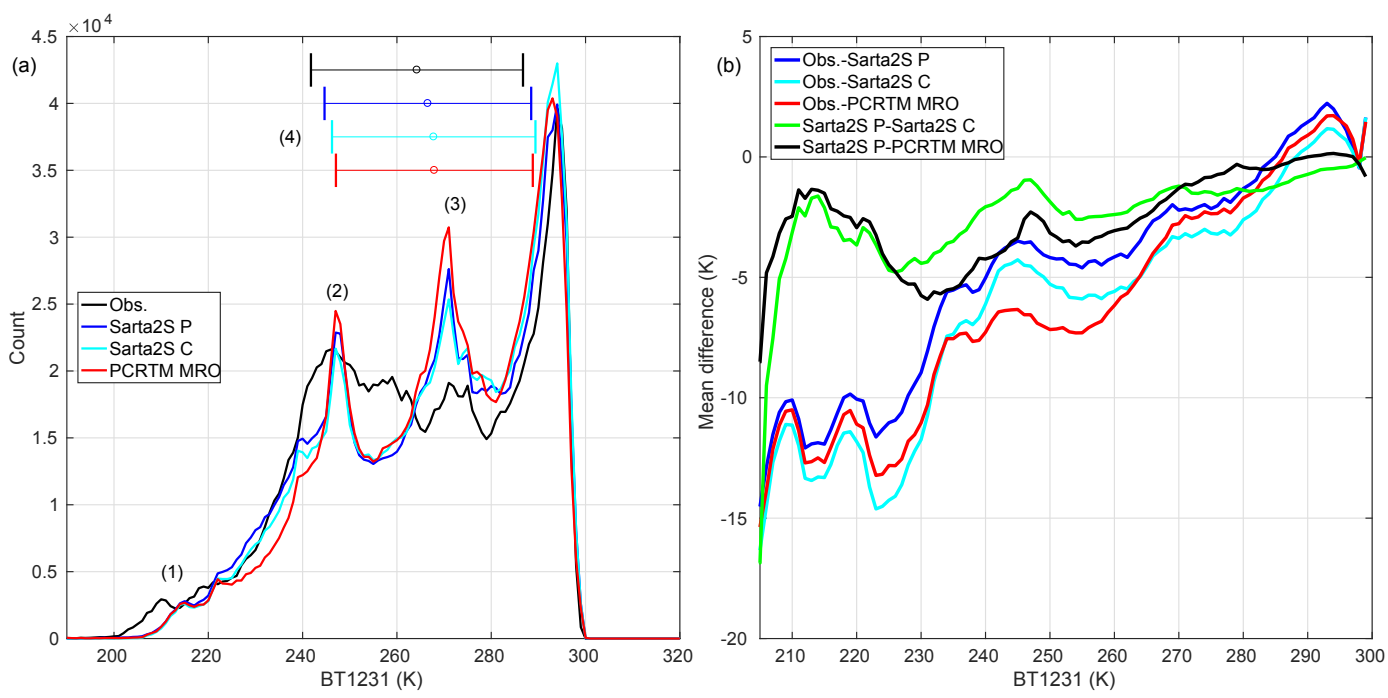

Figure 6. (a) The $1231 \mathrm{~cm}^{-1}$ channel brightness temperatures PDFs (probability distribution functions) for night scenes (global). Locations (1),(2) and (3) highlight large differences that are discussed in the text, while the horizontal lines and associated circles in location (4) represent the BT1231 $\mathrm{cm}^{-1}$ means and standard deviations. (b) Mean biases as a function of scene temperature for various RTAs tested here. Typical standard deviations between the different RTAs are about $10 \mathrm{~K}$, while the typical standard deviations between observations and calculations vary from $30 \mathrm{~K}$ (cold scenes) to $10 \mathrm{~K}$ (warm scenes).

Table 1. Night land and ocean $1231 \mathrm{~cm}^{-1}$ biases for 11 March $2011 \mathrm{in} \mathrm{K}$. "P" and "C" denote the cloud slabs placed at the peak (P) of the weighting function and the cloud profile centroid (C) respectively. Stemp, surface temperature; 2S, TwoSlab.

\begin{tabular}{|c|c|c|c|c|c|c|c|}
\hline Region & Stemp - Obs. & $\begin{array}{r}\text { Obs. - } \\
\text { PCRTM-MRO }\end{array}$ & $\begin{array}{r}\text { Obs. - } \\
\text { SARTA-2S (P) }\end{array}$ & $\begin{array}{r}\text { Obs. }- \\
\text { SARTA-2S (C) }\end{array}$ & $\mathrm{MRO}-2 \mathrm{~S}(\mathrm{P})$ & $\mathrm{MRO}-2 \mathrm{~S}(\mathrm{C})$ & $2 S(P)-2 S(C)$ \\
\hline Global & $12.5 \pm 14.3$ & $-3.69 \pm 10.4$ & $-2.3 \pm 11.0$ & $-3.6 \pm 10.8$ & $1.4 \pm 4.9$ & $0.1 \pm 4.5$ & $-1.2 \pm 4.5$ \\
\hline Tropical & $15.4 \pm 17.9$ & $-3.1 \pm 13.7$ & $-1.9 \pm 14.1$ & $-4.0 \pm 13.7$ & $1.3 \pm 5.3$ & $-1.0 \pm 4.0$ & $-2.2 \pm 4.0$ \\
\hline N. midlatitudes & $12.8 \pm 13.6$ & $-3.9 \pm 9.3$ & $-2.6 \pm 9.7$ & $-3.8 \pm 9.9$ & $1.3 \pm 4.6$ & $0.1 \pm 4.3$ & $-1.2 \pm 4.3$ \\
\hline S. midlatitudes & $14.4 \pm 12.7$ & $-4.2 \pm 9.5$ & $-3.5 \pm 9.9$ & $-5.4 \pm 9.6$ & $0.7 \pm 4.4$ & $-1.2 \pm 3.5$ & $-1.9 \pm 3.5$ \\
\hline N. polar & $6.9 \pm 9.4$ & $-2.9 \pm 7.1$ & $-1.2 \pm 7.8$ & $-0.3 \pm 8.1$ & $1.7 \pm 4.5$ & $2.6 \pm 5.2$ & $0.9 \pm 5.2$ \\
\hline S. polar & $9.9 \pm 9.2$ & $-4.9 \pm 6.5$ & $-2.8 \pm 8.0$ & $-3.5 \pm 7.3$ & $2.1 \pm 5.0$ & $1.4 \pm 4.3$ & $-0.7 \pm 4.3$ \\
\hline
\end{tabular}

and cyan) and PCRTM-MRO calculations are in red. While the tropical PDFs are quite similar between observations and both RTAs, the midlatitudes suggest ECMWF produces more clouds than observed. The polar PDF calculations are again quite similar, but with even large disagreements with observations. Accounting for this in detail is not the focus of the paper, but some insight was gained by comparing the cloud fields in Granule 001 obtained over the Southern Ocean-Antarctica (SOA) against those in Granule 137, which was filled with marine boundary layer (MBL) clouds off the coast of Namibia. For the SOA granule the mean CIWC and CLWC were $1 \times 10^{-5}$ and $0.3 \times 10^{-5} \mathrm{~g} \mathrm{~g}^{-1}$ with peaks centered at $825 \pm 50$ and $875 \pm 50 \mathrm{mb}$ respectively and mean cloud fractions of 0.3 at about $800-900 \mathrm{mb}$ and less than 0.1 above that. Conversely, the mean CIWC amounts for the MBL granule were almost 20 times larger at roughly the same vertical position (while the CIWC amounts were 10 times lower and cloud fractions were about the same); however, the computed radiances were about $5-10 \mathrm{~K}$ cooler than the surface temperatures, in rough agreement with the observations. The mean surface pressure and temperature for the SOA granule was $985 \mathrm{mb}$ and $273 \mathrm{~K}$ respectively, while the mean atmospheric temperature at $850 \mathrm{mb}$ was $262 \mathrm{~K}$. The bottom-most panel of Fig. 7 shows the observed peak was close to $260 \mathrm{~K}$ compared to the computed peak at about $270 \mathrm{~K}$. All this evidence points to one of two possibilities about the polar over-ocean clouds in the NWP model inability to statistically reproduce the observations. They could either be at too low an altitude or they could be at the right altitude and either have low optical thickness or a low cloud fraction.

\subsection{Spectral comparisons}

Finally we compare the observed and calculated spectra for ocean scenes where the ECMWF model sea surface temperature (SST) is very accurate and the emissivity is well known. As in Fig. 7, we divide these observations into tropical, mid- 


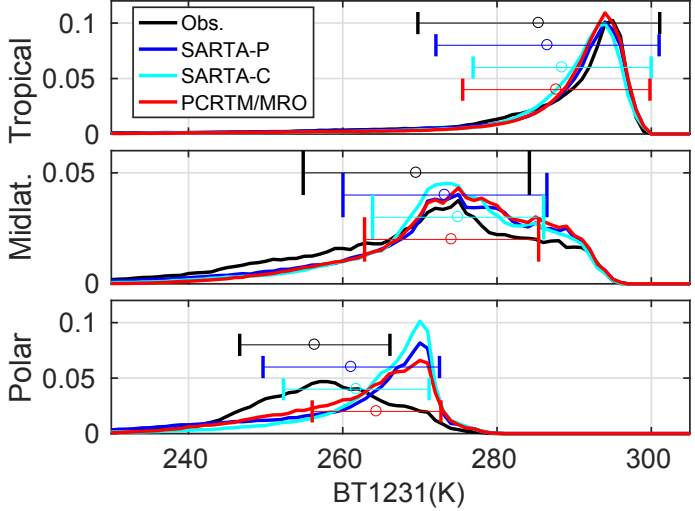

Figure 7. Brightness temperature PDFs for the night non-frozen ocean $1231 \mathrm{~cm}^{-1}$ channel for the data used in Fig. 8 separated tropical, midlatitude and polar regions. The whiskers correspond to the brightness temperature means and standard deviations.

latitude and polar zones (using boundaries at \pm 30 and \pm 60 ), with over 200000 observations per zone. Regions with sea ice contamination (according to ECMWF) were removed to avoid uncertainties in emissivity for the simulations. As with the PDF plots, the spatial mismatches between modeled and actual clouds will still lead to significant differences between observations and calculations, as seen in Fig. 4.

These comparisons are summarized in Fig. 8. In each panel the blue, green and red refer to the tropical, midlatitude and polar regions; the solid lines (marked S) refer to the SARTA-TwoSlab calculations, while the lines with " $x$ " (marked P) refer to PCRTM-MRO calculations. Note in this figure the label (PMRO) refers to PCRTM-MRO, and the SARTA calculations all use peak cloud weighting.

The mean tropical SARTA-TwoSlab and PCRTM-MRO calculations are slightly warmer than the observations, partially due to fewer and warmer deep convective clouds in the ECMWF model. The midlatitude window region calculations are on average about $5 \mathrm{~K}$ warmer than the observations. The calculations for the polar regions are noticeably warmer than the polar observations, with the SARTATwoSlab and PCRTM-MRO clouds simulations much more similar to each other than to the observations.

As expected from studying the $1231 \mathrm{~cm}^{-1}$ PDFs, the largest differences between the observations and calculations are in the polar (red) region. The tropical biases were typically the smallest, though their standard deviation is the largest (as the models and calculations have to span warm surface temperatures all the way to cold DCC tops). The largest spectral biases are in the window regions, which is to be expected as cloud effects are most readily seen in these regions.

The above plots are similar to all-sky monthly global averaged biases seen in (Shahabadi et al., 2016). They are put into context by considering the cloud forcing effect, which we simply define as the mean BT1231 difference between clear- sky calculations and observations. In the tropics, midlatitudes and polar regions they are 7.4, 10.2 and $12.6 \mathrm{~K}$ respectively. Conversely, the PCRTM-MRO versus SARTA-TwoSlab differences are a factor of 10 smaller, at $-0.78,-0.78$ and $1.89 \mathrm{~K}$ respectively. Again our main emphasis here is to validate the accuracy of our simple SARTA-TwoSlab algorithm relative to the more rigorous PCRTM-MRO RTA, not to evaluate the accuracy of the ECMWF model clouds.

\section{Application of TwoSlab code: all-sky retrieval}

One full AIRS granule's (6 min, 12150 spectra) worth of allsky retrievals using the SARTA-TwoSlab code is provided here using the OEM, which provides natural diagnostics such as DOF and AK that are extremely helpful in understanding the information content of our retrieval approach in the presence of highly variable clouds. This is a proof-of-concept retrieval which has been separately tested on several days' worth of AIRS observations. While considerable efforts have been put into selection of various regularization matrices and channels, we anticipate additional fine-tuning in the future.

\subsection{OEM approach}

We follow normal OEM notation here, where the observation vector $y(v)$ (brightness temperature) is modeled by the radiative forward model operator $F$ and $\epsilon(v)$ is the combined instrument and forward model noise:

$y(v)=F(x, v)+\epsilon(v)$,

where $v$ is the wavenumber and $x$ is the thermodynamic and cloud state. The solution is regularized with matrix $\mathbf{R}$ using a cost function $J$ (Rodgers, 2000; Steck, 2001) given by

$$
\begin{aligned}
J= & (y-F(x))^{T} \mathbf{S}_{\epsilon}^{-1}(y-F(x)) \\
& +\left(x-x_{\mathrm{a}}\right)^{T} \mathbf{R}(x)^{-1}\left(x-x_{\mathrm{a}}\right)+J_{\mathrm{sat}} .
\end{aligned}
$$

The first two terms are the observation and background penalties respectively, while the last is a constraint to reduce the amount of humidity supersaturation (Phalippou, 1996; Deblonde and English, 2003). Our regularization matrix contains both empirical regularization (Tikhonov) and a priori covariance-based terms. $J$ is minimized using a nonlinear Gauss-Newton iterative approach (Rodgers, 2000; Steck, 2001):

$$
\begin{aligned}
x_{n+1}= & x_{n}+\left(\mathbf{K}^{T} \mathbf{S}_{\epsilon}^{-1} \mathbf{K}+\mathbf{R}^{-1}\right)^{-1}\left(\mathbf{K}^{T} \mathbf{S}_{\epsilon}^{-1}\left(y-F\left(x_{n}\right)\right)\right. \\
& \left.-\mathbf{R}^{-1}\left(x_{n}-x_{a}\right)-J_{\text {sat }}{ }^{\prime}\left(x_{n}\right)\right),
\end{aligned}
$$

where $\mathbf{K}$ is the Jacobian. At present the observations covariance matrix $\mathbf{S}_{\epsilon}^{-1}$ is diagonal, combining a linear sum of forward model error $(\leq 0.2 \mathrm{~K}$ per channel) and AIRS channeldependent noise error. The retrieval currently uses no AIRS shortwave channels (past $2000 \mathrm{~cm}^{-1}$ ) but uses almost the same 500 longwave channels used in the AIRS L2 retrieval. 

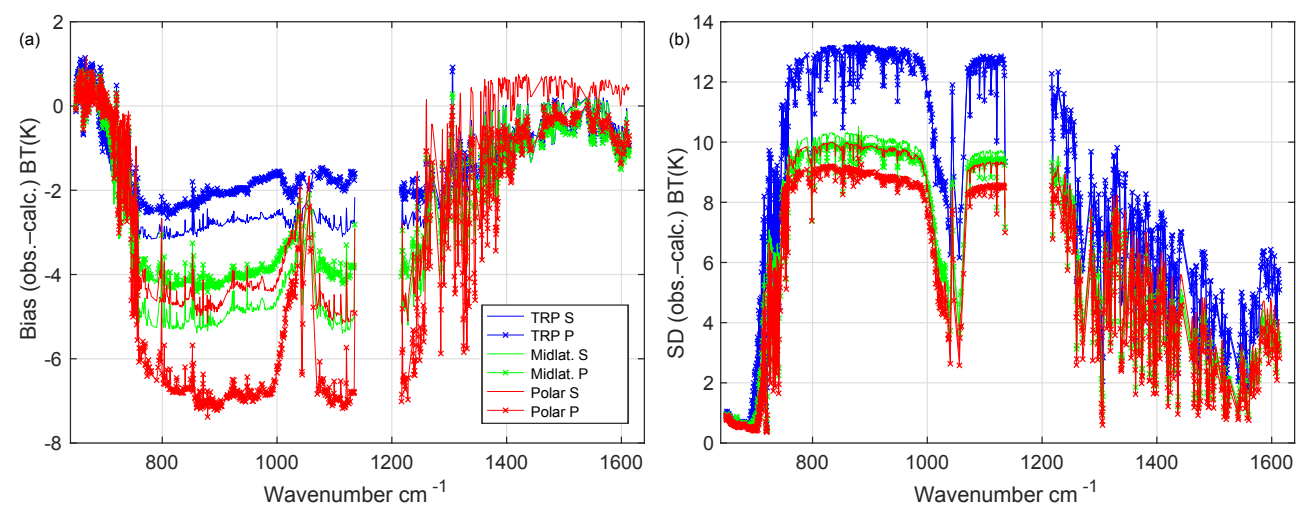

Figure 8. Mean biases (a) and standard deviations (b) between observations and calculations for ocean night scenes on 11 March 2011 for tropical (TRP), midlatitude (Midlat.) and polar (Polar) regions. Curves with $\times$ symbols are for PCRTM-MRO (PMRO). Solid lines denote SARTA-TwoSlab. Results are similar in the tropics and midlatitudes, with larger differences at the poles. See Fig. 7 for the corresponding BT1231 PDF plot.

\subsubsection{State vectors and OEM parameters}

The state vector consists of surface and profile temperatures (Kelvin), the logarithm of the water vapor profile (molecules $\mathrm{cm}^{-2}$ ), and a (logarithmic) multiplier for the ozone profile and two slab cloud amounts. The Jacobian matrix $\mathbf{K}$ in Eq. (5) is built using these parameters. For any FOV the iterations were halted after $i_{\max }=5$ iterations or, if there was no improvement in the $\chi^{2}$, after iteration $i \leq i_{\max }$.

The regularization matrix $\mathbf{R}$ is a block diagonal for the temperature and water vapor profiles and is a linear combination of altitude-dependent covariance matrices (with exponential decaying off-diagonal elements) and an $L_{1}$-type firstderivative Tikhonov smoother. Diagonal terms were added for the remaining state variables being retrieved. The humidity saturation penalty is of the form $J_{\text {sat }}=\sum_{i=1}^{i=N} J_{i}$, where $J_{i}$ is set to 0 if the relative humidity (RH) of the $i$ th layer is less than $100 \%$; otherwise, it is computed from $r\left(\log _{10} \frac{\mathrm{RH}(i)}{100}\right)^{3}$, where $r=100.0$.

For this paper we start with a smoothed climatological profile and use $2 \mathrm{~K}$ uncertainties for the temperature profiles, $0.3 \mathrm{~K}$ for the surface temperature, $60 \%$ uncertainty for water vapor profiles and $10 \%$ cloud loading uncertainty. We start with ECMWF surface temperatures since they are likely to be better than climatology. Land and ocean surface emissivity is set from a database (see Masuda et al., 1988; Zhou et al., 2011).

\subsubsection{The a priori retrieval}

The highly nonlinear effect of clouds on infrared radiative transfer makes retrieval success highly dependent on an accurate linearization point for cloud parameters. This fact has made it difficult to create physically based (not statistical) robust single-footprint infrared hyperspectral retrievals. Since typical infrared all-sky retrievals only have $2-4$ degrees of freedom for cloud fields (see Appendix B), it is essential that the linearization point and a priori covariances for clouds be as accurate as possible. Fortunately, NWP model forecasts such as from ECMWF provide reasonably accurate cloud fields derived using the best physics possible for an operational model. However, as we have shown earlier, perfect spatial placement of model clouds is not possible, and winds can move forecast clouds significantly during the $\pm 1.5 \mathrm{~h}$ time difference between observations and an ECMWF analysis and forecast.

The ECMWF cloud fields are statistically quite accurate in the sense that they reproduce similar spatial distributions of window channel brightness temperatures as the AIRS observations. Our approach is to compare the observed window channel brightness temperatures to those we compute from the ECMWF model (and cloud) fields using our TwoSlab approximation. We then find the closest spatial match between a particular window region observation and nearby simulated window channel radiance (in a least squares sense). The cloud fields from the ECMWF grid point with the closest matching radiance are then used as the linearization point for the retrieval for the AIRS scene. We only retrieve cloud loading after the linearization, while keeping particle effective size, cloud top and bottom pressure, and cloud fractions unchanged.

For example, Fig. 5 shows the overall spatial agreement between observed and simulated clouds over the tropical western Pacific; the ECMWF cloud fields are often offset from the observations (and are a factor of 2 fewer). The $300 \mathrm{hPa}$ ECMWF $(u, v)$ wind fields for this granule suggest that these convective regions are moving westward at approximately $36 \mathrm{~km} \mathrm{~h}^{-1}$. Since the forecast is approximately $0.8 \mathrm{~h}$ previous to the AIRS overpass, the model clouds could move by up to $30 \mathrm{~km}$ after the model forecast, or roughly two AIRS FOVs. Thus, just the time delays between forecast and observations can contribute to the cloud mismatch. 


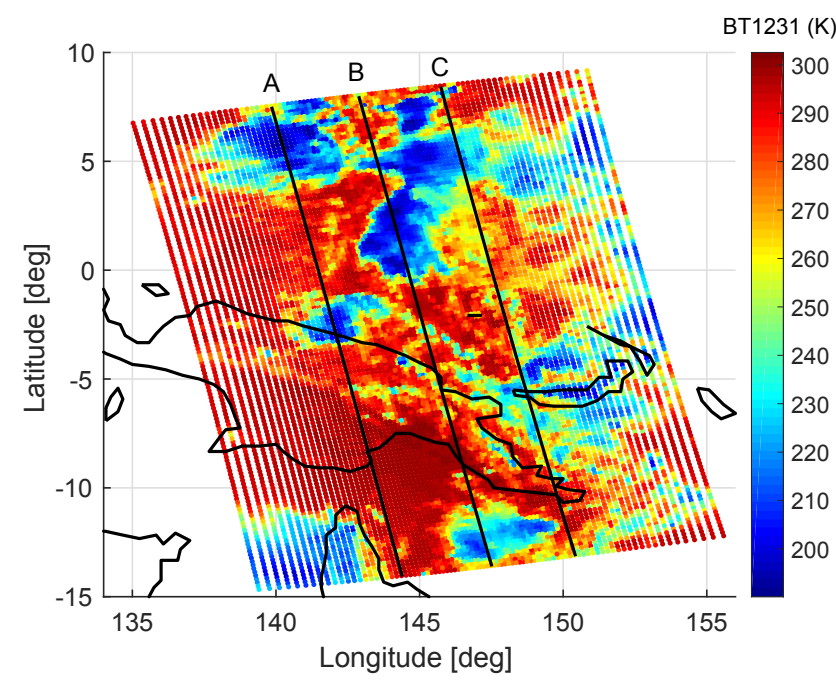

Figure 9. The $1231 \mathrm{~cm}^{-1}$ observed brightness temperatures for Granule 039 on 11 March 2011.

We point out that the thermodynamic fields from ECMWF $3 \mathrm{~h}$ forecasts (and/or analysis) are nearly identical to global radiosonde measurements (see for example the figures in Sect. 3 of Ingleby, 2017) and would also be an ideal starting point for retrieval of the temperature and humidity profiles. However, for this proof-of-concept paper the temperature and water vapor profile linearization point and a priori is instead taken from a climatology in order to more easily demonstrate the performance of the retrieval algorithm and the cloud and thermodynamic information contained in the AIRS radiances. The climatology came from 10-year co-located monthly averaged AIRS L3 fields (March 20042013). The water vapor a priori uncertainty was set to $60 \%$ and the temperature uncertainty to $2 \mathrm{~K}$ while the surface temperature used a $0.3 \mathrm{~K}$ uncertainty.

If a single-footprint retrieval was used for production of a long time series of AIRS level 2 products, we believe a reanalysis (such as the ECMWF reanalysis, ERA; or the Modern-Era Retrospective Analysis for Research and Applications, MERRA; Gelaro et al., 2017) would be a more suitable a priori thermodynamic profile in that it would provide accurate profile estimates below thick clouds. The OEM framework will naturally provide this capability since the degrees of freedom of the retrieval shrink rapidly as the cloud thickness increases.

\subsection{Single-granule case study}

This section focuses on AIRS Granule 039 from 11 March 2011, a day scene over the tropical western Pacific containing many DCCs. Figure 9 maps the $1231 \mathrm{~cm}^{-1}$ BT observations. The three lines marked (A), (B) and (C) are at AIRS scan angles of roughly $-23,0$ and $+24^{\circ}$. Figure 10 shows the observations (black), retrievals (red) and original

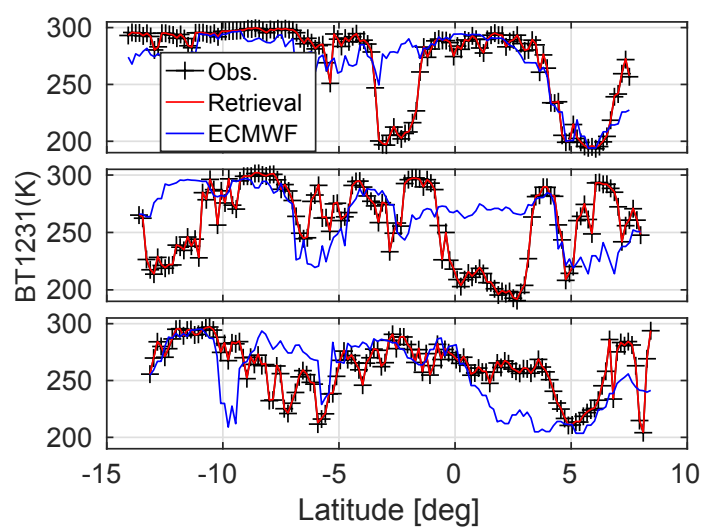

Figure 10. Three cross sections corresponding to the black lines in the granule image are shown. The curves are BT1231 $\mathrm{cm}^{-1}$ comparisons between the observations (black) and ECMWF (blue), which show the hit or miss characteristics of the spatial placement of the model clouds. The red curve just shows that we can find and include nearby clouds in the ECMWF model that have similar brightness temperatures to what is observed.

ECMWF calculations (blue) for these three lines. The significant offsets in the original (ECMWF) clouds are apparent by comparing the blue and black curves. The red curve shows how well the final retrieval simulates the window temperatures, due to a combination of moving the ECMWF cloud fields to the appropriate pixels together with the adjustment of the cloud fields during the retrieval. The final calculations largely reproduce the observations. A more thorough examination of (longitude, latitude) versus BT1231 scatter plots after the retrieval show that the spatial patterns (including the cold DCC) correlate extremely well with the patterns seen in Fig. 9.

\subsubsection{Spectral biases and DOF}

A first step in testing retrieval performance is to examine the final retrieval residuals and their standard deviations shown in Fig. 11. Also shown are the initial differences between allsky radiances simulated with ECMWF (clouds in their original positions) and the AIRS observations (blue curve). Most of the bias relative to ECMWF is due to clouds and some significant water vapor differences. Our retrieval BT bias and standard deviation are given by the red curve. The standard deviations from the original ECMWF co-located cloud fields have been multiplied by 0.2 to fit on the graph. Most channel biases are in the $0.1-0.2 \mathrm{~K}$ range although larger biases are evident in the $650-700 \mathrm{~cm}^{-1}$ stratospheric region. Further work is needed to determine the cause of these deviations. We have included all retrievals here, including those with low degrees of freedom where the a priori thermodynamic profile is weighted heavily.

The retrieval standard deviations are far smaller than those for ECMWF in the region of strong water lines past 
$1400 \mathrm{~cm}^{-1}$. In this region cloud cover is unimportant except for the deepest DCCs, so this reduction in the standard deviations indicates skill in the retrievals. This is discussed in more detail below.

Figure 12 maps retrieval DOFs for this granule. Comparisons to Fig. 9 show the clear correlation between low window channel BT, due to clouds, and low retrieval DOFs. The DOFs range from 5 for thick DCCs (ice cloud loadings of $100+\mathrm{g} \mathrm{m}^{-2}$ ) and increase to larger than 10 for nearly clear scenes. These DOF values are more than a factor of 2 smaller than what is reported in Appendix B, where a diagonal-only error covariance matrix is used when assessing the $N$-level cloud information content. The red circles in the figure are the $60 \%$ of the observations in this granule where the L2 retrieval failed all the way down to the surface.

\subsubsection{Thermodynamic retrievals}

Temperature and water vapor retrieval statistics are shown in Figs. 13 and 14. Statistical measures for a single granule, especially in the tropics, are of limited value for understanding retrieval accuracy, especially for temperature. However, they do help indicate nominal performance and provide a measure of the impact of clouds on the DOFs and retrieval accuracy. In addition, although ECMWF is quite accurate globally, it is difficult to judge how well ECMWF represents the truth for a single granule but it is likely far better than our a priori thermodynamic climatology.

These statistics have been separated by the total number of DOFs in the retrieval. Figure 13 shows scenes with DOFs less than 7 (thick cloud, 77 FOVs) and while Fig. 14 has DOFs greater than 10 (almost or completely clear, 6220 FOVs). The small number of scenes with low DOFs also limit the utility of their statistics. The ECMWF and climatology profiles were multiplied by the retrieval averaging kernels for these comparisons.

The low-DOF case (Fig. 13) shows a smaller difference between retrieved profile temperature and a priori temperature in the lower troposphere, compared to the free and upper troposphere. This is not too surprising for a tropical granule. The cloud contamination that caused these low DOFs led the retrieval to stick to the a priori temperature in the troposphere. Small movements in the upper troposphere and stratosphere did occur that gave similar disagreements with the a priori temperature and ECMWF. The same is true for water vapor for the low-DOF case, any differences between the retrieval and either the a priori water vapor or ECMWF are in the upper troposphere, where the cloud contamination is lower.

The high-DOF cases in Fig. 14 indicate that the retrieval moved slightly away from the a priori temperature towards ECMWF at almost all levels. Results are a bit more mixed for water vapor. The retrieval standard deviation from ECMWF is lower than with the a priori water vapor or temperature in most of the troposphere.
A much more definitive diagnostic of retrieval performance is given in Fig. 15, which is a curtain plot of relative humidity along the line denoted by "B" in Fig. 9. Here we compare a series (from top to bottom) of our retrieval, ECMWF, the AIRS L2 retrieval and a priori humidity profiles, with the vertical axis being pressure and horizontal axis being latitude. The bottom panel is a plot of the BT $1231 \mathrm{~cm}^{-1}$ channel which is an excellent proxy for cloud top height and opacity. The a priori water vapor and ECMWF water vapor structures have been multiplied by the averaging kernels.

There is little water vapor structure in the a priori water vapor (fourth panel) given that it is a monthly average of many years. The retrieved water vapor (top panel) shows significant structure along this track, with some small instabilities in the regions of thick high clouds (near $-12^{\circ}$ latitude and $+6^{\circ}$ latitude); the black and red circles mark the positions of ice and water clouds, with the circle size representing the optical depth. The ECMWF water fields show very significant structure, which becomes quantitatively similar to our retrievals once the retrieval averaging kernels are applied to the ECMWF profiles. Overall the retrieved water vapor fields move from being almost structureless to showing similarities to those of ECMWF, indicating a very encouraging retrieval performance. For example both are relatively dry at $500 \mathrm{mb}$ between $-5^{\circ} \mathrm{S}$ and the equator, at approximately the same locations where AIRS L2 retrievals were drier. Overall our retrieval is showing higher humidity values than either L2 or ECMWF. In particular where the bottom panel shows medium clouds (for example at $-11,-3^{\circ} \mathrm{S}$ and $+6^{\circ} \mathrm{N}$ ) our retrieval shows high humidity at $400-600 \mathrm{mb}$, and where the bottom panel shows thick high cloud (for example between 0 and $+3^{\circ} \mathrm{N}$ ) the retrieval returns the a priori humidity.

The ability of the retrieval to catch some of the uppertroposphere variability near $150 \mathrm{hPa}$ seen in ECMWF indicates good vertical resolution as well. Note that the retrieval does not use any information from ECMWF, except for the clouds. We also comment that the upper troposphere and lower stratosphere (UTLS) humidity from the AIRS L2 is significantly lower than either ECMWF or our retrieval, with the blanked-out areas indicating where the surface AIRS L2 QA flags were bad.

The higher tropopause RH that was initiated by climatology remained unaffected by the retrieval; this could be alleviated by an improved first guess of the thermodynamic state, as well as choosing WV channels that peak very high in the atmosphere. In the future we plan to use a reanalysis as our a priori thermodynamic profiles, which will be adjusted by the retrieval when there are low to medium optically thick clouds. The use of a fixed shape for the ozone profile is also a limitation of the present retrieval that will be removed in later versions. 


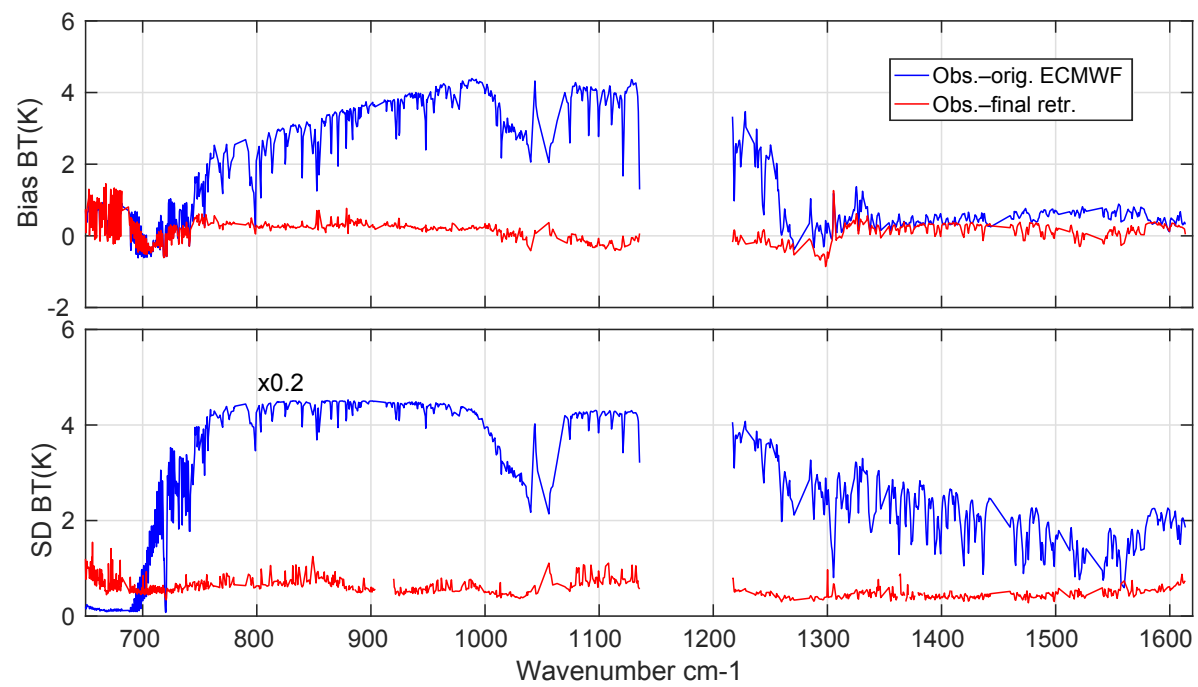

Figure 11. Retrieval results for Granule 039 shown in Fig. 9. The original biases between observations and ECMWF before the retrieval and cloud replacement are also shown (in blue); note that this standard deviation has been reduced by $5 \times$ for plotting.

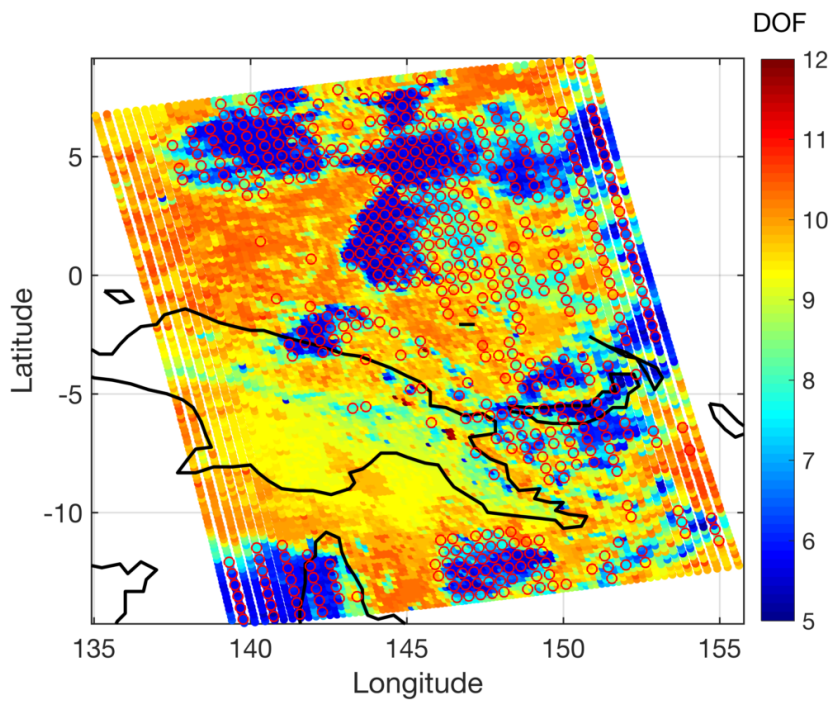

Figure 12. Retrieved degrees of freedom for Granule 039 show evident dependence on observed BT1231 (which depends on cloud loading). The red circles denote locations of the AIRS FORs (fields of regard) $(3 \times 3)$ where the retrieval quality down to the surface is 2, meaning missing or do not use; this happened for $60 \%$ of the L2 FORs.

\subsubsection{Cloud parameter changes}

Comparisons between the initial ECMWF TwoSlab cloud parameters (found by matching window BTs to nearby ECMWF scenes) and the retrieved cloud parameters have a number of understandable differences. It is well known that NWP models do not produce as many deep convective clouds as observed so it is understandable that the mean ice cloud fraction changed from less than 0.5 to higher values rang-

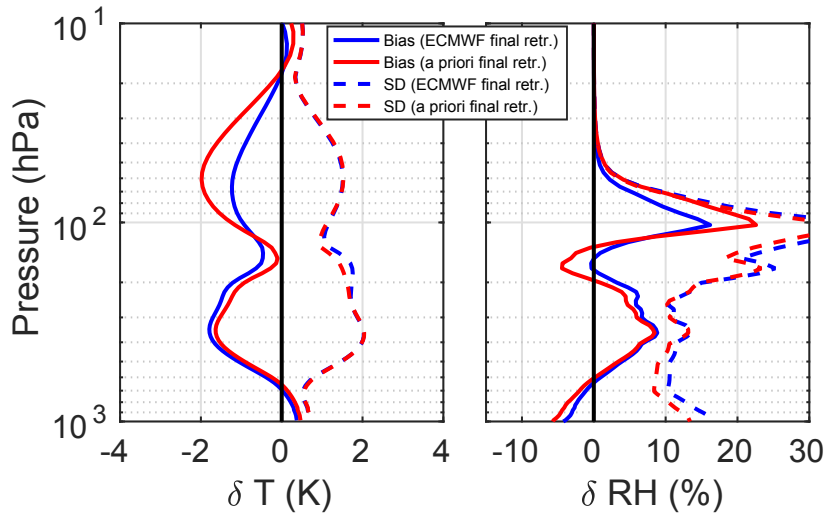

Figure 13. Retrieval statistics for Granule 039 for thick clouds, defined by the number of DOFs to be $<7$. Comparisons between ECMWF profiles (multiplied by averaging kernels) and our retrievals in blue, compared to a priori profiles (multiplied by averaging kernels) and our retrievals in red. The solids are the mean differences while the dashed are the standard deviations of the differences. Note that the results from our retrieval used over $95 \%$ of scenes in the granule while AIRS L2 had a $60 \%$ yield of good or best QA down to the surface; the intersection here is 77 profiles. Very similar plots are obtained if we use all 1592 low-DOF retrievals.

ing from 0.6 to 0.9 (these can be quite thin ice clouds). The water cloud fractions increased slightly from less than 0.3 and generally decreased for the higher factions. In addition, the frequency of high ice cloud tops (less than $250 \mathrm{mb}$ ) increased while the rest decreased; for water clouds the largest increase in frequency of occurrence was seen between 500 and $700 \mathrm{mb}$.

A quick validation of our ice cloud optical depths is achieved by comparing AIRS L2 ice optical depths versus 


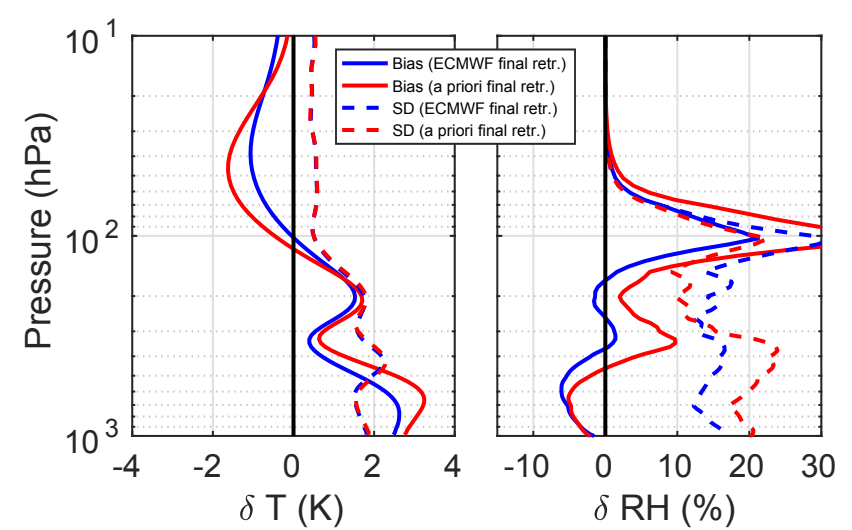

Figure 14. Same as Fig. 13 except now uses cases for thin clouds, defined by DOFs to be $>10 ; 6220$ profiles were used here (compared to 7296 if we ignore the AIRS L2 QA and only use high-DOF retrievals, again with very similar plots being obtained).

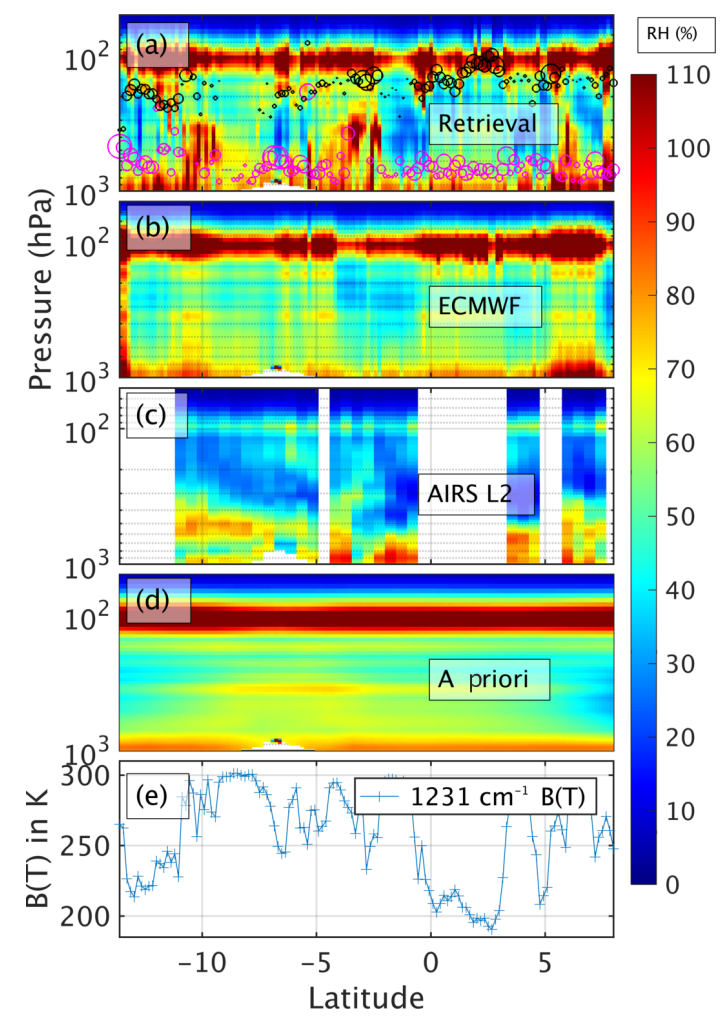

Figure 15. Comparing percent relative humidity (color bar) along track "B" in Fig. 9. From (a) to (e) we have our retrieval, ECMWF, the AIRS L2 retrieval and a priori humidity profiles. Panel (e) is a plot of the observed BT $1231 \mathrm{~cm}^{-1}$ channel. The black and red circles in (a) are the positions of ice and water clouds, with the circle size denoting the OD.

our retrieved ice cloud loading (in $\mathrm{g} \mathrm{m}^{-2}$ ). Figure 16 is a comparison of AIRS L2 ice optical depths (Kahn et al., 2014) versus our retrieved ice cloud loading (in $\mathrm{g} \mathrm{m}^{-2}$ ), clearly showing a proportional relationship. This is very encourag-

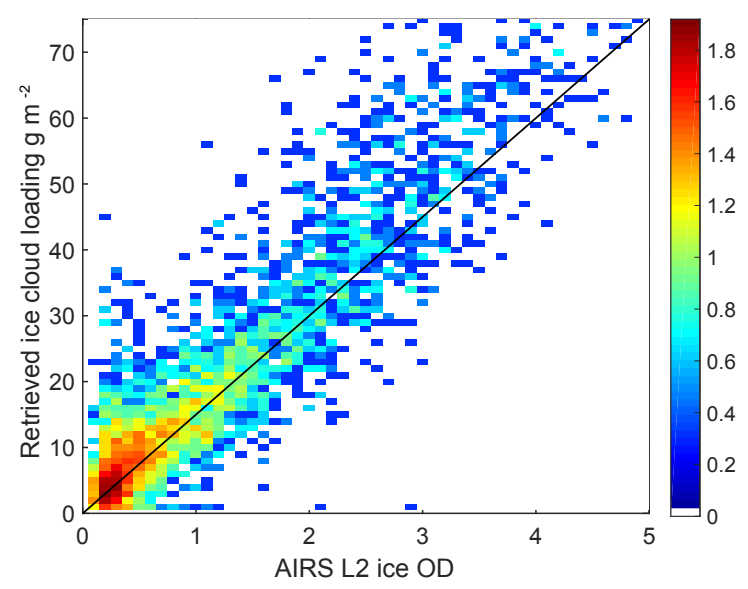

Figure 16. Retrieved ice cloud amount (in $\mathrm{g} \mathrm{m}^{-2}$ ), compared to AIRS L2 ice cloud optical depths. The color bar is the $\log (10)$ of the number of points in the bin.

ing in that we are also retrieving the full thermodynamic state and water cloud parameters simultaneously.

The retrieval used cloud heights derived from matching to nearby ECMWF cloud fields. Figure 17 provides partial validation of that approach by comparing Moderate Resolution Imaging Spectrometer (MODIS) L2 ice cloud heights with the initial ECMWF ice cloud heights and those used in the retrieval. MODIS is also on the Aqua platform and uses a mixture of infrared, near-infrared and visible channels to retrieve cloud optical properties at $10 \mathrm{~km}$ resolution. Here we used the "CloudTopHeight" product from the Collection 6 (MYDO6) dataset; see https://modis-atmos.gsfc.nasa. gov/_docs/C6MOD06OPUserGuide.pdf for details. We have masked the water clouds using the MODIS ice cloud phase product.

The left-hand panel shows that the ECMWF ice cloud placement north of the island of Papua New Guinea is at $8 \mathrm{~km}$ (light blue) with a number of high cloud tops straddling the topmost part of the granule; the center panel shows our algorithm moved the $8 \mathrm{~km}$ high clouds to be northeast of the island plus it placed some very high cold DCC tops almost on a line along $145^{\circ} \mathrm{E}$ longitude, which is consistent with what was retrieved by MODIS (right-hand panel). In addition, clouds over the island of Papua New Guinea were removed by our algorithm, which is consistent with what MODIS retrieved.

\section{Conclusions}

A fast infrared radiative transfer algorithm with the ability to handle two scattering layers (from clouds, aerosols, volcanic dust) has been described and compared to a more sophisticated and often slower approach (maximum random overlap). Our ultimate goal is to perform single-footprint retrievals with hyperspectral IR sounder radiances. In particu- 

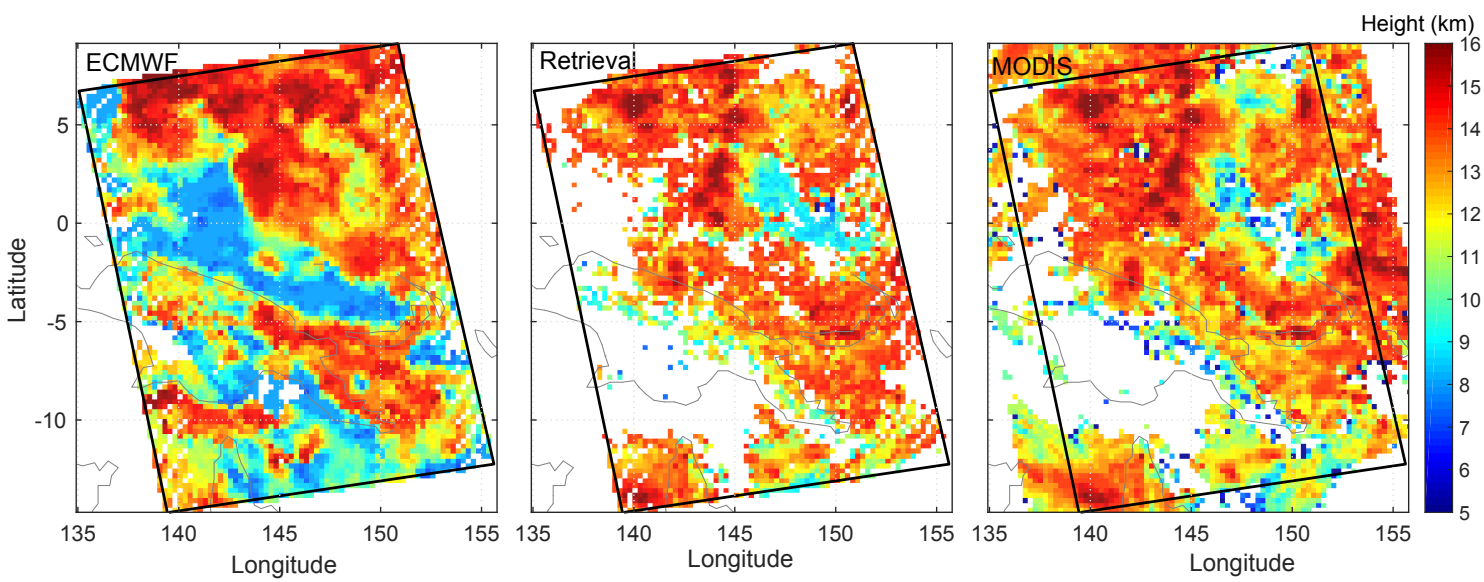

Figure 17. Comparisons between cloud top height: (left) original ECMWF ice cloud top; (center) ice cloud top heights used in the retrieval (ice clouds with optical depth $<0.5$ have been removed); (right) MODIS L2 ice cloud top heights; the black lines outline AIRS Granule 039 . It is clearly evident that the initial cloud tops from ECMWF were reset by our algorithm to closely resemble the MODIS-retrieved cloud tops; also note the similarity to the observed BT1231 in Fig. 9. Generally the low ECMWF cloud heights are associated with very low optical depths.

lar we wish to handle the very common case of two cloud layers (water, ice) in order to provide accurate, higher spatial resolution retrievals of temperature and water vapor (and other minor gases). This approach uses the observed radiances in the retrieval rather than derived equivalent cloudcleared radiances that are presently used for the NASA AIRS level 2 products. The complexity of true cloud structures cannot be retrieved with hyperspectral IR radiances, and we have shown that only a maximum of 2-4 cloud parameters can be derived from a single scene, suggesting that only a simple RTA is needed.

However, if the a priori cloud parameters are not sufficiently accurate, it can be very difficult for the retrieval to converge quickly, if at all. Our approach uses NWP model fields (here ECMWF) to initialize the cloud model fields. Four sub-columns (at most) are needed to compute a radiance for one scene, which is a small speed penalty in fast radiative transfer models, where most of the time is spent in computing the atmospheric optical depths. The TwoSlab model can be an order of magnitude faster than typical implementations of MRO and has nearly the same accuracy, both in terms of mean spectral radiances and radiance PDFs. The spectral bias between all-sky AIRS observations and calculations are dominated by spatial location mismatches between actual and forecast clouds. Both approaches used the ECMWF cloud fields, and in general both differed from observations similarly. For example, PCRTM-MRO is slightly more accurate in the tropics than SARTA-TwoSlab, while the opposite is true in polar regions. However, the comparisons of RTA simulations to observations are both limited by the accuracy of the NWP model fields and especially by small spatial mismatches between NWP and observed clouds. The larger er- rors of both RTA approaches in the polar regions indicate that ECMWF clouds have too-low optical depths.

We demonstrated the feasibility of the SARTA-TwoSlab approach by performing single-footprint retrievals using an AIRS tropical granule. Our approach to the retrieval cloud initialization and a priori thermodynamic and cloud parameters, which is key to the successful results shown here, was to use NWP cloud fields in the region of the footprint of interest based on matching simulated and observed radiances. These matched cloud fields are then converted from $N$-layer NWP cloud fields to the two-layer SARTA-TwoSlab. Together these steps provide a method for robust, and fast, retrievals from single-footprint all-sky hyperspectral spectra.

A major advantage of single-footprint retrieval using the OEM approach is the retrieval quality diagnostics that are provided within the OEM framework. We demonstrated that the retrieval DOFs are reduced in the presence of thicker clouds. However, we were able to reproduce much of the water vapor variability in ECMWF (assumed to be relatively accurate, partly because it agrees with our retrievals) when using a climatology for the water vapor and temperature a priori.

Existing AIRS L2 retrievals fail in scenes with thick clouds and where the 3-by-3 set of radiances used for cloud clearing are too homogeneous (which is not always the case in thick clouds as seen in Fig. 12). This is particularly troublesome for long-term climate studies in that the AIRS L2 sampling is incomplete and may alias certain climate variables, especially for water vapor where microwave retrievals (that are part of the AIRS L2 system) have a more limited value. 
(Smith et al., 2015) discusses climate change studies using the homogeneous geographic sampling resulting from single-footprint retrievals that are physically and statistically based (whereas ours are physically based, using an all-sky RTA through all the iterations). The retrieval approach examined here may be able to address sampling limitations of the existing AIRS retrievals by (1) using single-footprint retrievals that are not affected by cloud-clearing failures for highly uniform scenes and (2) using a priori thermodynamic profile information in a statistically correct way under conditions of thick clouds. A possible approach is to use a reanalysis for the a priori thermodynamic profiles rather than climatology in order to insert the best possible information in cases where the DOFs of the retrieval are very low.
This work does not represent a rigorous analysis of the accuracy of our retrieval approach, but only a proof of principle that the technique appears viable. In particular, the temperature retrievals are not stressed in a tropical environment, although our results suggest significant skill for water vapor. The retrieval tests shown here were mostly all over ocean where the surface emissivity is well known. Over land, we will need to include a variable surface emissivity into the retrieval. The time taken to retrieve one single footprint (at the 100-layer native SARTA vertical resolution) is on average under $2.5 \mathrm{~s}$, which includes matching the AIRS L1b radiances to climatology and NWP cloud fields and converting the NWP cloud profiles to slab clouds. This is very competitive with the official AIRS L2 product which takes about 1.5 second per field of regard using 20 trapezoid vertical functions (but does retrieve profiles of some additional trace gases and computes outgoing longwave radiation).

Data availability. Data used for this study are available at https://doi.org/10.5281/zenodo.1157936 (DeSouza-Machado et al., 2018). 


\section{Appendix A: Clear-sky retrieval comparisons with ECMWF}

Clear-sky biases are likely to arise from inaccuracies in the geophysical parameters, such as highly variable water vapor fields and surface temperatures. The radiance measured by and simulated for the $1231 \mathrm{~cm}^{-1}$ channel for clear-sky scenes over the oceans is dominated by the surface temperature, water vapor (which is very variable) and to a much lesser extent temperatures in the lower atmosphere; errors in any of these would affect the comparisons of observed versus simulated radiances. Using an OEM retrieval scheme (Rodgers, 2000) (also see Sect. 6), we investigated possible errors for NWP fields used in clear-sky scene calculations by using the AIRS thermal infrared window channels to retrieve tropical sea surface temperature and column water vapor (WV) amounts, as well as column $\mathrm{O}_{3}$ amount using the $10 \mu \mathrm{m}$ channels. Averaged over $\sim 10000+$ fields of view for day and for night, the nighttime ECMWF SST was adjusted by an offset of $-0.5 \pm 2.6 \mathrm{~K}$, while the column WV was adjusted by a multiplicative factor of $1.1 \pm 0.2$ and the column $\mathrm{O}_{3}$ was adjusted by a multiplicative factor of $1.2 \pm 0.05$. The corresponding daytime adjustments were $-0.3 \pm 0.8 \mathrm{~K}$ for nighttime ECMWF SST, $1.1 \pm 0.1$ for column WV and $1.12 \pm 0.05$ for column $\mathrm{O}_{3}$. While a discussion of the SST adjustments is outside the scope of the paper, the required reduction from the retrieval suggests there is some residual cloud leakage present in the AIRXBCAL clear-sky dataset.

\section{Appendix B: Information content of AIRS radiances}

A uniform mixing ratio ice cloud $\left(10 \times 10^{-6} \mathrm{~g} \mathrm{~g}^{-1}\right)$ from 200 to $440 \mathrm{mb}$ and a uniform mixing ratio water cloud $(1 \times$ $10^{-6} \mathrm{~g} \mathrm{~g}^{-1}$ ) from 440 to $900 \mathrm{mb}$ were inserted into a tropical profile spanning 97 AIRS layers (1013 mb to TOA). Finitedifference Jacobians for the temperature, humidity and cloud profiles were used to compute the degrees of freedom of the signal (Rodgers, 2000); AIRS NEDT values converted to BT noise levels were used for a diagonal noise $\mathbf{S}_{e}$ matrix. For this Appendix we use a diagonal $\mathbf{S}_{a}$ geophysical error matrix which had a $1.0 \mathrm{~K}$ temperature error and 0.1 fraction $(10 \%) \mathrm{WV}(z)$ error at all layers; similarly, we assumed a 0.1 fraction $(10 \%)$ error for $\operatorname{CIWC}(z), \operatorname{CLWC}(z)$ at all layers. The computed degrees of freedom of signal for $\mathrm{T}(z), \mathrm{WV}(z)$, $\operatorname{CLWC}(z)$ and $\operatorname{CIWC}(z)$ were $13.78,6.46,1.75$ and 2.45 respectively. The last two numbers imply that the information in the 100-layer cloud profiles can indeed be compactly represented by two parameters (cloud top, cloud amount). The corresponding numbers computed for a clear atmosphere are $[13.72,6.99,0,0]$, while those obtained for a thick ice cloud (DCC) and thick water cloud atmosphere are [7.89, 0.99, 0, 2.41] and [10.34, 3.52, 2.30, 0] respectively.

\section{Appendix C: Sensitivity analysis of the TwoSlab cloud scheme}

The SARTA-TwoSlab model has four parameters per cloud plus a cloud slab fraction and cloud slab overlap parameter that are derived from NWP model fields. The four parameters are the vertical placement and width of the slabs, the cloud loading (integrated CIWC or CLWC amounts) and the effective particle size. Since there are only 2-4 degrees of freedom for clouds in the spectra, for the retrieval only the cloud amounts were varied while the vertical placement, fraction and effective particle size were kept fixed, after the "closest" cloud was found.

Here we briefly explain the changes in the simulated radiances as the cloud vertical placements are changed. An observation dataset of 7377 AIRS observations from 1 March 2009 is used here, as it was chosen to provide maximum variability due to clouds, over land and ocean, and span all climate regions (personal communication, George Aumann, Jet Propulsion Laboratory, CA). The BT1231 $\mathrm{cm}^{-1}$ channel is used to study differences between observations and calculations. For the TwoSlab model two placings of the cloud slabs were studied - one where the slab was placed where the cloud's weighting function peaks and the other at the NWP cloud profile centroid.

As can be seen from the whisker plots of Figs. 6 (left panel) and 7 (all three sub-panels), especially when considering the mean and standard deviation, the MRO calculations are more similar to the TwoSlab centroid calculations than the TwoSlab peak calculations. This can be understood from the point of view of where the cloud radiates from: in the peak case we place the cloud higher up, which leads to colder calculations; in the centroid case one would expect the multiple sub-pixels of an MRO simulation to also radiate primarily from this region. A (finite-difference or analytic) Jacobian is easily computed using the slab clouds, while a Jacobian with the MRO representation would be computationally expensive and probably ill-defined as the sub-pixel cloud amounts and fractions are randomly determined at each stage of the calculation. On average, placing the clouds at the centroid globally displaces water clouds downwards by about $80 \mathrm{mb}$ (from $723 \mathrm{mb}$ ) and ice clouds downwards by about $60 \mathrm{mb}$ (from about $400 \mathrm{mb}$ ) from the peak cases. 
Competing interests. The authors declare that they have no conflict of interest.

Acknowledgements. We acknowledge the use of ECMWF model fields to compute radiances. The hardware used in the computational studies is part of the UMBC High Performance Computing Facility (HPCF). The facility is supported by the US National Science Foundation through the MRI program (grant nos. CNS0821258 and CNS-1228778) and the SCREMS program (grant no. DMS-0821311), with additional support from the University of Maryland, Baltimore County (UMBC). See www.umbc.edu/hpcf for more information on HPCF and the projects using its resources. Helpful discussions with George Aumann and Eric Maddy are gratefully acknowledged. Scott Hannon wrote the clear and scattering versions of SARTA and helped develop the TwoSlab clouds.

Edited by: Andrew Sayer

Reviewed by: two anonymous referees

\section{References}

Allan, R., Slingo, A., Milton, S., and Culverwell, I.: Exploitation of Geostationary Earth Radiation Budget data using simulations from a numerical weather prediction model: Methodology and data validation, J. Geophys. Res., 110, D14111, https://doi.org/10.1029/2004JD005698, 2005.

August, T., Klaes, D., Schlussel, P., Hultberg, T., Crapeau, M., Arriaga, A., O'Carroll, A., Coppens, D., Munro, R., and Calbet, X.: IASI on Metop-A : Operational Level 2 retrievals after 5 years in orbit, J. Quant. Spectrosc. Ra., 113, 1340-1371, https://doi.org/10.1016/j.jqsrt.2012.02.028, 2012.

Aumann, H. and Pagano, T.: First light results from AIRS on EOS AQUA, in: Proceedings of the SPIE Conference 5548-42, Optical Science and Technology, Crete, 2002.

Aumann, H., Broberg, S., Elliot, D., Gaiser, S., and Gregorich, D.: Three years of AIRS radiometric calibration validation using sea surface temperatures, J. Geophys. Res., 111, 2156-2202, https://doi.org/10.1029/2005JD006822, 2006.

Bauer, P., Auligne, T., Bell, W., Geer, A., Guidard, V., Heilliette, S., Kazumori, M., Kim, M.-J., Liu, E., McNally, A., MacPherson, B., Okamato, K., Renshaw, R., and Riishojgaard, L.-P.: Satellite cloud and precipitation assimilation at operational NWP centres, Q. J. Roy. Meteorol. Soc., 137, 1934-1951, https://doi.org/10.1002/QJ.905, 2011.

Baum, B., Yang, P., Heidinger, A., Heymsfield, A., Li, J., and Nasiri, S.: Bulk scattering properties for ice clouds. Part 3. High resolution spectral models from 100 to $3250 \mathrm{~cm}^{-1}$, J. Appl. Meteor. Clim., 46, 423-434, 2007.

Baum, B., Yang, P., Heymsfield, A., Schmitt, C., Xie, Y., Bansemer, A., Hu, Y.-X., and Zhang, Z.: Improvements to shortwave bulk scattering and absorption models for the remote sensing of ice clouds, J. Appl. Meteor. Clim, 50, 1037-1056, 2011.

Chen, X., Huang, X., and Liu, X.: Non-negligible effects of cloud vertical overlapping assumptions on longwave spectral fingerprinting studies, J. Geophys. Res., 118, 7309-7320, https://doi.org/10.1002/jgrd.50562, 2013.
Chou, M.-D., Lee, K.-T., Tsay, S.-C., and Fu, Q.: Parameterization for Cloud Longwave Scattering for use in Atmospheric Models, J. Climate, 12, 159-169, 1999.

Clarisse, L., Hurtmans, D., Prata, A., Karagulian, F., Clerbaux, C., De Maziere, M., and Coheur, P.-F.: Retrieving radius, concentration, optical depth, and mass of different types of aerosols from high-resolution infrared nadir spectra, Appl. Opt, 49, 3712-3722, https://doi.org/10.1364/A0.49.003713, 2010.

Clough, S., Shephard, M., Mlawer, E., Delamere, J., Iacono, M. J., Cady-Pereira, K., Boukabara, S., and Brown, P.: Atmospheric radiative transfer modeling: a summary of the AER codes, J. Quant. Spectrosc. Ra., 91, 233-244, https://doi.org/10.1016/j.qsrt2004.05.058, 2005.

De Souza-Machado, S., Strow, L. L., Motteler, H., and Hannon, S.: kCARTA: An Atmospheric Radiative Transfer Algorithm using Compressed Lookup Tables, Tech. rep., University of Maryland Baltimore County, Department of Physics, available at: http://asl. umbc.edu/pub/packages/kcarta.html (last access: January 2018), 2002.

De Souza-Machado, S., Strow, L. L., Motteler, H., Hannon, S., Lopez-Puertas, M., Funke, B., and Edwards, D.: Fast Forward Radiative Transfer Modeling of 4.3 um Non-Local Thermodynamic Equilibrium effects for the Aqua/AIRS Infrared Temperature Sounder, Geophys. Res. Lett., 34, L01802, https://doi.org/10.1029/2006GL026684, 2007.

De Souza-Machado, S., Strow, L. L., Imbiriba, B., McCann, K., Hoff, R., Hannon, S., Martins, J., Tanré, D., Deuzé, J., Ducos, F., and Torres, O.: Infrared retrievals of dust using AIRS: comparisons of optical depths and heights derived for a North African dust storm to other collocated EOS A-Train and surface observations, J. Geophys. Res., 115, D15201, https://doi.org/10.1029/2009JD012842, 2010.

DeSouza-Machado, S., L. L. Strow, A. Tangborn, X. Huang, X. Chen, X. Liu, W. Wu, and Q. Yang: Dataset for AMT-2017-261 by DeSouza-Machado et al., available at: https://doi.org/10.5281/zenodo.1157936, 2018.

Deblonde, G. and English, S.: 1D Variational Retrievals from SSMIS Simulated Observations, J. App. Met., 42, 1406-1420, 2003.

Dee, D. P., Uppala, S. M., Simmons, A. J., Berrisford, P., Poli, P., Kobayashi, S., Andrae, U., Balmaseda, M. A., Balsamo, G., Bauer, P., Bechtold, P., Beljaars, A. C. M., van de Berg, L., Bidlot, J., Bormann, N., Delsol, C., Dragani, R., Fuentes, M., Geer, A. J., Haimberger, L., Healy, S. B., Hersbach, H., Hólm, E. V., Isaksen, L., Kållberg, P., Köhler, M., Matricardi, M., McNally, A. P., Monge-Sanz, B. M., Morcrette, J.-J., Park, B.-K., Peubey, C., de Rosnay, P., Tavolato, C., Thépaut, J.-N., and Vitart, F.: The ERA-Interim reanalysis: configuration and performance of the data assimilation system, Q. J. Roy. Meteorol. Soc., 137, 553597, 2011.

EUMETSAT: IAI Level 2: Product Generation Specification, Tech. rep., EUMETSAT, available at: http://www.eumetsat.int/ website/home/Data/TechnicalDocuments/index.html Reference EPS.SYS.SPE.990013 8E, 2016.

Gambacorta, A.: The NOAA Unique CrIS/ATMS Processing System (NUCAPS): Algorithm Theoretical Basis Documentation, Tech. rep., NCWCP, available at: http://www.ospo.noaa.gov/Products/atmosphere/soundings/ 
nucaps/docs/NUCAPS_ATBD_20130821.pdf (last access: January 2018), 2013.

Gelaro, R., McCarty, W., Suárez, M. J., Todling, R., Molod, A., Takacs, L., Randles, C. A., Darmenov, A., Bosilovich, M. G., Reichle, R., Wargan, K., Coy, L., Cullather, R., Draper, C., Akella, S., Buchard, V., Conaty, A., da Silva, A. M., Gu, W., Kim, G., Koster, R., Lucchesi, R., Merkova, D., Nielsen, J. E., Partyka, G., Pawson, S., Putman, W., Rienecker, M., Schubert, S. D., Sienkiewicz, M., and Zhao, B.: MERRA-2 Overview: The Modern-Era Retrospective Analysis for Research and Applications, Version 2 (MERRA-2), J. Clim., 30, 5419-5454, https://doi.org/10.1175/JCLI-D-16-0758.1, 2017.

Griessbach, S., Hoffman, L., Hopfner, M., Riese, M., and Spang, R.: Scattering in infrared radiative transfer: A comparison between the spectrally averaging model JURASSIC and the line-by-line model KOPRA, J. Quant. Spectrosc. Ra., 127, 102-118, 2013.

Hess, M., Koepke, P., and Schult, I.: Optical Properties of Aerosols and Clouds: The Software Package OPAC, B. Am. Meteorol. Soc., 79, 831-844, 1998.

Huang, H.-L., Yang, P., Wei, H., Baum, B., Hu, Y., Antonelli, P., and Ackerman, S.: Inference of ice cloud properties from highspectral resolution infrared observation, IEEE T. Geosci. Remote, 42, 842-852, 2004.

Ingleby, B.: An assessement of different radiosonde types 2015/2016, ECMWF Technical Memorandum, 807, 1-71, 2017.

Irion, F. W., Kahn, B. H., Schreier, M. M., Fetzer, E. J., Fishbein, E., Fu, D., Kalmus, P., Wilson, R. C., Wong, S., and Yue, Q.: Single-footprint retrievals of temperature, water vapor and cloud properties from AIRS, Atmos. Meas. Tech. Discuss., https://doi.org/10.5194/amt-2017-197, in review, 2017.

Kahn, B., Eldering, A., Clough, S., Fetzer, E., Fishbein, E., Gunson, M., Lee, S.-Y., Lester, P., and Realmuto, V.: Near micron sized cirrus cloud particles in high-resolution infrared spectra : an orographic case study, Geophys. Res. Lett., 30, 1441, https://doi.org/10.1029/2003GL016909, 2003.

Kahn, B., Eldering, A., Fetzer, E., Fishbein, E., Lee, S.-Y., Liou, K., DeSouza-Machado, S., Strow, L., and Hannon, S.: Nighttime cirrus detection using the Atmospheric Infrared Sounder window channels and total column water vapor, J. Geophys. Res., 110, D07203, https://doi.org/10.1029/2004JD005430, 2005.

Kahn, B. H., Irion, F. W., Dang, V. T., Manning, E. M., Nasiri, S. L., Naud, C. M., Blaisdell, J. M., Schreier, M. M., Yue, Q., Bowman, K. W., Fetzer, E. J., Hulley, G. C., Liou, K. N., Lubin, D., Ou, S. C., Susskind, J., Takano, Y., Tian, B., and Worden, J. R.: The Atmospheric Infrared Sounder version 6 cloud products, Atmos. Chem. Phys., 14, 399-426, https://doi.org/10.5194/acp-14-3992014, 2014.

King, M., Platnick, S., Menzel, P., Ackerman, S., and Hubanks, P.: Spatial and Temporal Distribution of Clouds Observed by MODIS Onboard the Terra and Aqua Satellite, IEEE, 51, 38263852, https://doi.org/10.1109/TGRS.2012.2227333, 2013.

Klein, S. and Jakob, C.: Validation and sensitivities of frontal clouds simulated by the ECMWF model, Mon. Weather Rev., 127, 2514-2531, 1999.

Liu, X., Smith, W., Zhou, D., and Larar, A.: Principal component based radiative transfer model for hyperspectral sensors: theoretical concepts, Appl. Opt., 45, 201-209, 2006.

Liu, X., Zhou, D. K., Larar, A. M., Smith, W. L., Schluessel, P., Newman, S. M., Taylor, J. P., and Wu, W.: Retrieval of atmospheric profiles and cloud properties from IASI spectra using super-channels, Atmos. Chem. Phys., 9, 9121-9142, https://doi.org/10.5194/acp-9-9121-2009, 2009.

Liuzzi, G., Masiello, G., Serio, C., Meloni, D., Di Biagio, C., and Formenti, P.: Consistency of dimensional distributions and refractive indices of desert dust measured over Lampedusa with IASI radiances, Atmos. Meas. Tech., 10, 599-615, https://doi.org/10.5194/amt-10-599-2017, 2017.

Masuda, K., Takashima, T., and Takayama, Y.: Emissivity of pure and sea waters for the model sea surface in the infrared window regions, Remote Sens. Environ., 24, 313-329, 1988.

Matricardi, M.: The inclusion of aerosols and clouds in RTIASI, the ECMWF fast radiative transfer model for the infrared atmospheric sounding interferometer, ECMWF Technical Memorandum, 474, 1-55, 2005.

McClatchey, R., Fenn, R., Selby, J., Volz, F., and Garing, J.: Optical properties of the atmosphere, Tech. Rep. AFCRL-72-0497, AFGL(OPI), Hanscom AFB, MA 01731, 1972.

Niu, J., Yang, P., Huang, H.-L., Davies, J., Li, J., Baum, B. A., and $\mathrm{Hu}$, Y.: A fast infrared radiatbe transfer model for overlapping clouds, J. Quant. Spectrosc. Ra., 103, 447-459, 2007.

$\mathrm{Ou}$, S.-C. and Liou, K.: Ice microphysics and climatic temperature feedback, Atmos. Res., 35, 127-138, 1995.

Ou, S.-C., Kahn, B., Liou, K., Takano, Y., Schreier, M., and Yue, Q.: Retrieval of Cirrus Cloud Properties From the Atmospheric Infrared Sounder: The k-Coefficient Approach Using CloudCleared Radiances as Input, IEEE, 51, 1010-1024, 2013.

Phalippou, L.: Variational Retrieval of humidity profile, windspeed and cloud liquid water path with the SSM/I : Potential for Numerical Weather Prediction, Q. J. Roy. Meteorol. Soc., 122, 327-355, 1996.

Reale, O., Lau, K., Susskind, J., and Rosenberg, R.: AIRS impact on analysis of an extreme rainfall event (Indus River, Valley, Pakistan 2010) with a global data assimilation and forecast system, J. Geophys. Res., 117, D08103, https://doi.org/10.1029/2011JD017093, 2012.

Rodgers, C.: Inverse Methods for Atmospheric Sounding, World Scientific, Singapore, 2000.

Rossow, W. and Schiffer, R.: The International Satellite Cloud Climatology Project (ISCCP): The First Project of the World Climate Research Programme, B. Am. Meteorol. Soc., 64, 779-784, 1983.

Rossow, W. and Schiffer, R.: ISCCP Cloud Data Products, B. Am. Meteorol. Soc., 72, 2-20, 1991.

Saunders, R., Rayer, P., Brunel, P., von Engeln, A., Borman, N., Strow, L., Hannon, S., Helilliette, S., Liu, X., Miskolczi, F., Han, Y., Masiello, G., Moncet, J.-L., Uymin, G., SHerlock, V., and Turner, D.: A intercomparison of radiative transfer models for simulating Atmospheric Infrared Sounder radiances, J. Geophys. Res., 112, D01S90, https://doi.org/10.1029/2006JD007088, 2007.

Segelstein, D.: The complex refractive index of water, Master's thesis, University of Missouri, Kansas City, 1981.

Shahabadi, M., Huang, Y., Garand, L., Heillette, S., and Yang, P.: Validation of a weather forecast model at radiance level against satellite observations allowing quantification of temperature, humidity, and cloud-related biases, J. Adv. Model. Earth Syst., 8, 1453-1467, https://doi.org/10.1002/2016MS000751, 2016. 
Smith, N., Smith, W., Weisz, E., and Revercomb, H.: AIRS, IASI, and CrIS retrieval records at climate scales: An investigation into the propagation of systematic uncertainty, J. Appl. Met. Climl., 54, 1465-1481, 2015.

Stamnes, K., Tsay, S.-C., Wiscombe, W., and Jayaweera, K.: Numerically Stable Algorithm for discrete ordinate method Radiative Transfer in multiple scattering and emitting layered media, Appl. Opt., 27, 2502-2509, 1988.

Steck, T.: Methods for determining regularization for Atmospheric Retrieval Problems, Appl. Opt., 41, 1788-1797, 2001.

Strow, L., Hannon, S., DeSouza-Machado, S., Tobin, D., and Motteler, H.: An Overview of the AIRS Radiative Transfer Model, IEEE T. Geosci. Remote Sens., 41, 303-313, 2003.

Strow, L., Hannon, S., DeSouza-Machado, S., Tobin, D., and Motteler, H.: Validation of the Version 4 AIRS Radiative Transfer Algorithm, J. Geophys. Res., 111, https://doi.org/10.1029/2005JD006008, 2006.

Susskind, J., Barnet, C., and Blaisdell, J.: Atmospheric and Surface Parameters from Simulated AIRS/AMSU/HSB Sounding Data: Retrieval and Cloud Clearing Methodology, Adv. Space. Sci, 21, 369-384 https://doi.org/10.1016/S0273-1177(97)00916-2, 1998.

Susskind, J., Barnet, C., and Blaisdell, J.: Retrieval of atmospheric and surface parameters from AIRS/AMSU/HSB data under cloudy conditions, IEEE T. Geosci. Remote Sens., 41, 390409, 2003.

Uppala, S., Kallberg, P., and Simmons, A.: The ERA-40 re-analysis, Q. J. Roy. Meteorol. Soc., 131, 2961-3011, 2005.

Vidot, J., Baran, A., and Brunel, P.: A new ice cloud parameterization for infrared radiative transfer simulation of cloudy radiances: Evaluation and optimization with IIR observations and ice cloud profile retrieval products, J. Geophys. Res., 120, 69376951, https://doi.org/10.1002/2015JD023462, 2015.
Wei, H., Yang, P., Li, J., Baum, B., Huang, H., Platnick, S., Hu, Y., and Strow, L.: Retrieval of semitransparent ice cloud optical thickness from Atmospheric Infrared Sounder (AIRS) measurements, IEEE T. Geosci. Remote Sens., 42, 2254-2267, 2004.

Weisz, E., Smith, W., and Smith, N.: Advances in simultaneous atmospheric profile and cloud parameter regression based retrieval from high-spectral resolution radiance measurements, J. Geophys. Res., 118, 6433-6443, https://doi.org/10.1002/jgrd.50521, 2013.

Wu, D., Ackerman, S., Davies, R., Diner, D., Garay, M., Kahn, B., Maddux, B., Moroney, C., Stephens, G., Veefkind, J., and Vaughan, M.: Vertical distributions and relationships of cloud occurence frequency as observed by MISR, AIRS, MODIS, OMI, CALIPSO and CloudSat, Geophys. Res. Lett., 36, L09821, https://doi.org/10.1029/2009GL037464, 2009.

Wu, W., Liu, X., Zhou, D., Larar, A., Yang, Q., Kizer, S., and Liu, Q.: The Application of PCRTM Physical Retrieval Methodology for IASI Cloudy Scene Analysis, IEE, 99, 1-15, https://doi.org/10.1109/TGRS.2017.2702006, 2017.

Yang, P., Gao, B., Baum, B., Hu, Y., Wiscombe, W., Tsay, S.-C., and Winker, D. M., and Nasiri, S.: Radiative properties of cirrus clouds in the infrared (8-13 microns), J. Quant. Spectrosc. Ra., 70, 473-504, 2002.

Zhou, D., W.L., S. S., Liu, X., Larar, A., Huang, H.-L., Li, J., McGill, M., and Mango, S.: Thermodynamic and cloud parameter retrieval using inrared spectral data, Geophys. Res. Lett., 32, L15805, https://doi.org/10.1029/2005GL023211, 2005.

Zhou, D., Larar, A., Liu, X., Smith, W., Strow, L., Yang, P., Schlüssel, P., and Calbet, X.: Global Land Surface Emissivity Retrieved From Satellite Ultraspectral IR Measurements, IEEE Trans. Geosci. Remote Sens., 49, 1277-1290, 2011. 